\title{
Molecular gas in the northern nucleus of Mrk 273: Physical and chemical properties of the disc and its outflow
}

\author{
R. Aladro ${ }^{1,2}$, S. König ${ }^{2}$, S. Aalto ${ }^{2}$, E. González-Alfonso ${ }^{3}$, N. Falstad ${ }^{2}$, S. Martín ${ }^{4,5}$, S. Muller ${ }^{2}$, S. García-Burillo ${ }^{6}$, \\ C. Henkel ${ }^{1,7}$, P. van der Werf ${ }^{8}$, E. Mills ${ }^{9}$, J. Fischer ${ }^{10}$, F. Costagliola ${ }^{2}$, and M. Krips ${ }^{11}$
}

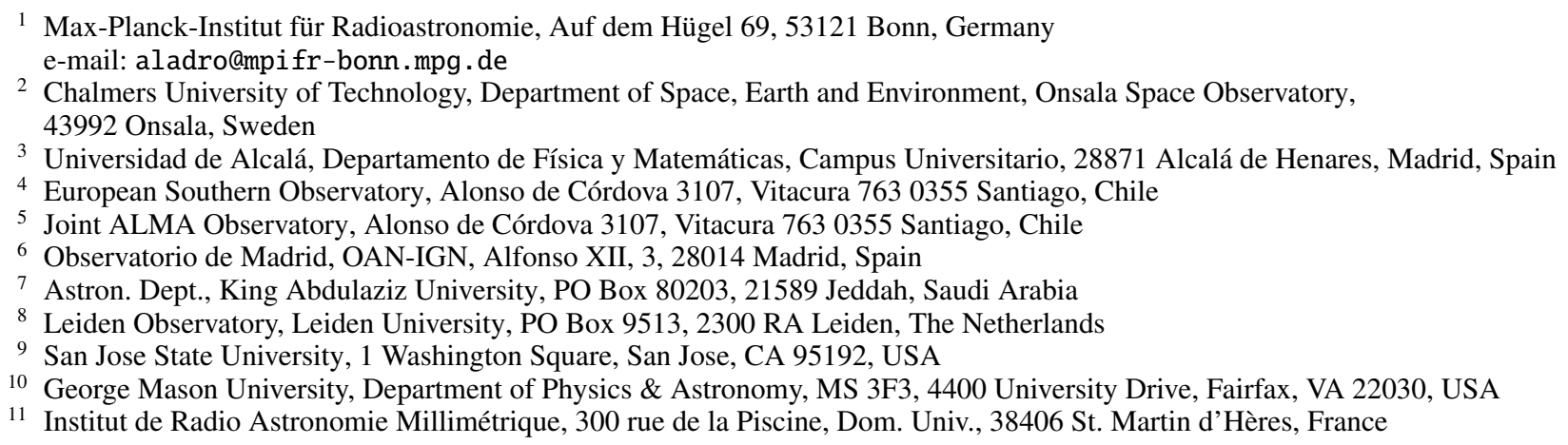

Received 2 May 2018 / Accepted 25 May 2018

\begin{abstract}
Aiming to characterise the properties of the molecular gas in the ultra-luminous infrared galaxy Mrk 273 and its outflow, we used the NOEMA interferometer to image the dense-gas molecular tracers $\mathrm{HCN}, \mathrm{HCO}^{+}, \mathrm{HNC}, \mathrm{HOC}^{+}$and $\mathrm{HC}_{3} \mathrm{~N}$ at $\sim 86 \mathrm{GHz}$ and $\sim 256 \mathrm{GHz}$ with angular resolutions of $4 \prime \prime 9 \times 44^{\prime \prime} 5(\sim 3.7 \times 3.4 \mathrm{kpc})$ and $0^{\prime \prime} .61 \times 00^{\prime \prime} 55(\sim 460 \times 420 \mathrm{pc})$. We also modelled the flux of several $\mathrm{H}_{2} \mathrm{O}$ lines observed with Herschel using a radiative transfer code that includes excitation by collisions and far-infrared photons. The disc of the Mrk 273 north nucleus has two components with decoupled kinematics. The gas in the outer parts $(R \sim 1.5 \mathrm{kpc})$ rotates with a south-east to north-west direction, while in the inner disc $(R \sim 300 \mathrm{pc})$ follows a north-east to south-west rotation. The central $300 \mathrm{pc}$, which hosts a compact starburst region, is filled with dense and warm gas, and contains a dynamical mass of $(4-5) \times 10^{9} M_{\odot}$, a luminosity of $L_{\mathrm{HCN}}^{\prime}=(3-4) \times 10^{8} \mathrm{~K} \mathrm{~km} \mathrm{~s}^{-1} \mathrm{pc}^{2}$, and a dust temperature of $55 \mathrm{~K}$. At the very centre, a compact core with $R \sim 50 \mathrm{pc}$ has a luminosity of $L_{\mathrm{IR}}=4 \times 10^{11} L_{\odot}$ ( $30 \%$ of the total infrared luminosity), and a dust temperature of $95 \mathrm{~K}$. The core is expanding at low velocities $\sim 50-100 \mathrm{~km} \mathrm{~s}^{-1}$, probably affected by the outflowing gas. We detect the blue-shifted component of the outflow, while the red-shifted counterpart remains undetected in our data. Its cold and dense phase reaches fast velocities up to $\sim 1000 \mathrm{~km} \mathrm{~s}^{-1}$, while the warm outflowing gas has more moderate maximum velocities of $\sim 600 \mathrm{~km} \mathrm{~s}^{-1}$. The outflow is compact, being detected as far as $460 \mathrm{pc}$ from the centre in the northern direction, and has a mass of dense gas $\leq 8 \times 10^{8} M_{\odot}$. The difference between the position angles of the inner disc $\left(\sim 70^{\circ}\right)$ and the outflow $\left(\sim 10^{\circ}\right)$ indicates that the outflow is likely powered by the AGN, and not by the starburst. Regarding the chemistry in Mrk 273, we measure an extremely low $\mathrm{HCO}^{+} / \mathrm{HOC}^{+}$ratio of $10 \pm 5$ in the inner disc of Mrk 273.
\end{abstract}

Key words. galaxies: individual: Mrk 273 - galaxies: nuclei - ISM: molecules - line: profiles - astrochemistry galaxies: kinematics and dynamics

\section{Introduction}

Tracers of dense molecular gas are good probes of the central regions of active galaxies, where molecular regions are subjected to strong radiation fields (X-rays, cosmic rays, and ultraviolet (UV) fields) created by massive star formation and/or active galactic nuclei (AGNs). In particular, the rotational transitions of $\mathrm{HCN}$ and $\mathrm{HCO}^{+}$have bright emission and high dipole moments (hence large critical density) and are therefore convenient tracers of the dense gas component in galactic centres.

In the particular case of ultra-luminous infrared galaxies (ULIRGs), the nuclei are embedded in large quantities of gas and dust produced by merging processes and massive star formation. In these conditions, the visual extinctions can be as high as $A v>1000$ mag when the $\mathrm{H}_{2}$ column densities exceed $>10^{24} \mathrm{~cm}^{-2}$ in very compact regions of only a few pc (e.g. Costagliola et al. 2015; Aalto et al. 2017). In these extremely obscured environs, even the millimetre $(\mathrm{mm}) / \mathrm{sub}-\mathrm{mm}$ emission can be significantly attenuated by self- or continuum absorption, therefore probing only the gas emitted at distances $\geq 100$ pc from the central engines (e.g. Aalto et al. 2015b; Martín et al. 2016). Complementing $\mathrm{mm} / \mathrm{sub}-\mathrm{mm}$ observations with radiatively pumped molecular lines emitted in the infrared (IR), where most of the ULIRGs luminosity is emitted, allows us to probe the regions of the dusty cores more deeply. In particular, $\mathrm{H}_{2} \mathrm{O}$ lines are excited via absorption and re-emission of IR photons produced very close to the central engines, and therefore provide essential information about the physical conditions of the hidden power sources of ULIRGs.

Mrk $273(\mathrm{~F} 13428+5608)$ is a ULIRG $\left(L_{\mathrm{IR}}=1.3 \times 10^{12} L_{\odot}\right.$, Gao et al. 1999) located at a distance of $157 \mathrm{Mpc}\left(V_{\mathrm{LSR}}^{\mathrm{optical}}=\right.$ $\left.11339 \mathrm{~km} \mathrm{~s}^{-1}, z=0.037780,1^{\prime \prime}=761 \mathrm{pc}^{1}\right)$. Its complex morphology reflects a recent merger event between two or more galaxies.

1 https://ned.ipac.caltech.edu 
Near-infrared (NIR), radio emission, and HI images show two nuclei separated by $\sim 1^{\prime \prime}$ in the northeast-southwest direction (hereafter the northern and southern nucleus) plus a weaker third source that might be another nucleus or a starburst region triggered by the merger (Majewski et al. 1993; Cole et al. 1999; Condon et al. 1991). The nature of the progenitors has been the subject of several studies that claim either AGN or starburst activities. Mrk 273 is classified as a Seyfert 2 galaxy in the optical and NIR (U et al. 2013; Rodríguez Zaurín et al. 2014; Iwasawa et al. 2018), having an AGN bolometric luminosity of $\log \left(L_{\mathrm{AGN}}\right)=44.73 \mathrm{erg} \mathrm{s}^{-1}$, and a ratio between the bolometric luminosity of the AGN and the total bolometric luminosity of the galaxy of $L_{\mathrm{AGN}} / L_{\mathrm{Bol}}=0.08$ (Nardini et al. 2009). Nevertheless, far infrared (FIR) and $\mathrm{mm}$ data point to a compact ultra-luminous starburst region in the northern nucleus (Condon et al. 1991; Majewski et al. 1993; Downes \& Solomon 1998). The total star formation rate (SFR) of the galaxy is $139 M_{\odot} \mathrm{yr}^{-1}$ (Cicone et al. 2014).

Molecular observations reveal a complex structure in the centre of Mrk 273. Carbon monoxide maps show extended gas streamers in the north-south direction, a nuclear disc (of $2^{\prime \prime}$ size) oriented east-west, and a very compact core $\left(0.35^{\prime \prime} \times 0.2^{\prime \prime}\right)$ containing a powerful starburst (Downes \& Solomon 1998). All these components belong to the northern nucleus (which is the strongest radio source), while there is no evidence of significant amounts of molecular gas in the southern objects.

A cool molecular outflow has been detected in Mrk 273 using $\mathrm{H}_{2}, \mathrm{CO}$, and $\mathrm{OH}$ observations (U et al. 2013; Veilleux et al. 2013; Cicone et al. 2014; González-Alfonso et al. 2017). From $\mathrm{CO}(1-0)$ observations, the outflow appears clearly in both line wings at high velocities $|v|>400 \mathrm{~km} \mathrm{~s}^{-1}$, and extends from the northern nucleus about $600 \mathrm{pc}$ to the north. The CO channel-velocity maps also show a low velocity component $\left(v>150 \mathrm{~km} \mathrm{~s}^{-1}\right)$ of the red-shifted outflow expanding to the north. The total mass of the outflow estimated from $\mathrm{CO}$ is $\sim 2 \times 10^{8} M_{\odot}$.

The multi-phase composition of the outflow has been revealed by infrared and optical observations. Hydroxide $(\mathrm{OH})$ detections (Veilleux et al. 2013; González-Alfonso et al. 2017) probe a more compact and warmer phase of the outflow that expands at velocities of $100-700 \mathrm{~km} \mathrm{~s}^{-1}$. This component travels shorter distances $(160 \mathrm{pc})$ before it presumably cools down, and its mass and mass-loss rate are $5 \times 10^{7} M_{\odot}$ and $120 M_{\odot} \mathrm{yr}^{-1}$. Colina et al. (1999) detected an ionised component of the outflow by using the [OIII] $\lambda=5007 \AA$ optical line. The hot, ionised gas goes much further, as far as $\pm 5^{\prime \prime}(\sim 3.8 \mathrm{kpc})$ along the northsouth direction, and reaches velocities as high as $\pm 1200 \mathrm{~km} \mathrm{~s}^{-1}$. Other IR and optical lines, namely $\mathrm{H}_{2}, \mathrm{HeI}, \mathrm{Br} \gamma$, and [CII], also show the low-velocity component of the outflow $\left( \pm 200 \mathrm{~km} \mathrm{~s}^{-1}\right)$, as well as a moderate velocity component $(600 \pm 300) \mathrm{km} \mathrm{s}^{-1}$ heading towards the north (U et al. 2013; Janssen et al. 2016).

Despite being one of the most luminous ULIRGs in the local universe, molecular detections towards Mrk 273 were still scarce and limited to $\mathrm{CO}, \mathrm{OH}$, and $\mathrm{H}_{2}$. In this paper we present observations of molecules detected for the first time in this galaxy in the $\mathrm{mm} / \mathrm{sub}-\mathrm{mm}$ and FIR ranges. In Sect. 2 we present our observations with the NOEMA and Herschel telescopes and the data reduction. The continuum and spectroscopic data analyses are presented in Sect.3, where we also describe our modelling of $\mathrm{H}_{2} \mathrm{O}$. The asymmetric, double-peaked line profiles of the inner disc are discussed in Sect.4.1. The detection of the Mrk 273 outflow and its properties are presented in Sect. 4.2. A brief discussion of the expansion of the galaxy core can be found in Sect. 4.3. The non-detection of vibrationally excited $\mathrm{HCN}$ and $\mathrm{HC}_{3} \mathrm{~N}$ emission is addressed in Sect. 4.4. We also discuss the $\mathrm{HCN} / \mathrm{HNC}, \mathrm{HCN} / \mathrm{HCO}^{+}$, and $\mathrm{HCO}^{+} / \mathrm{HOC}^{+}$ brightness temperature ratios (Sect.4.5). The different origin of $\mathrm{HOC}^{+}$, the only species not peaking at the very centre, is discussed in Sect. 4.6. Finally, our conclusions are summarised in Sect. 5.

\section{Observations and data reduction}

\subsection{NOEMA}

The $\mathrm{HCN}, \mathrm{HCO}^{+}, \mathrm{HNC}(1-0)$ and $\mathrm{HC}_{3} \mathrm{~N}(10-9)$ lines were observed simultaneously with the NOEMA interferometer on April 7, 2015 (with seven antennae), and June 12 and 15, 2017 (with eight antennae during a total time of $8.9 \mathrm{~h}$ (precipitable water vapour (pwv) 5-9 mm), while the $\mathrm{HCN}, \mathrm{HCO}^{+}$and $\mathrm{HOC}^{+}(3-2)$ transitions were observed, also simultaneously, for $5.6 \mathrm{~h}$ ( $\mathrm{pwv} \sim 1-2 \mathrm{~mm}$ ). The receivers were tuned to centre their lower side bands at $85.8 \mathrm{GHz}(\lambda \sim 3 \mathrm{~mm})$ and $257.5 \mathrm{GHz}$ $(\lambda \sim 1 \mathrm{~mm})$, respectively. The receivers were connected to the WideX correlator and provided a $3.6 \mathrm{GHz}$ bandwidth in two orthogonal polarisations (which were averaged). The data were calibrated and imaged with CLIC and MAPPING within the GILDAS' $^{\prime}$ package ${ }^{2}$. Source 3C84 was used as a bandpass calibrator, and the phase and flux calibrations were done by observing 1418+546 and MWC349, respectively.

The observations were centred on $\mathrm{RA}(\mathrm{J} 2000)=$ $13^{\mathrm{h}} 44^{\mathrm{m}} 42 \mathrm{~s} 1, \operatorname{Dec}(\mathrm{J} 2000)=55^{\circ} 53^{\prime} 13^{\prime \prime} \cdot 5$. The original spectral resolutions of $6.8 \mathrm{~km} \mathrm{~s}^{-1}(3 \mathrm{~mm})$ and $2.3 \mathrm{~km} \mathrm{~s}^{-1}(1 \mathrm{~mm})$ were smoothed to $68-70 \mathrm{~km} \mathrm{~s}^{-1}$. The final rms of the cubes, averaged across the spectral channels that do not contain line emission, are $0.3 \mathrm{mJy}$ beam $^{-1}(3 \mathrm{~mm})$ and $1.3 \mathrm{mJy} \mathrm{beam}^{-1}$ $(1 \mathrm{~mm})$. We used a natural weighting mode and 0":8 (for the $3 \mathrm{~mm}$ data) and 0 '.05 (for the $1.3 \mathrm{~mm}$ data) pixel sizes to create the data cubes, and the Hogbom deconvolution method to clean them. The sizes of the primary beams were 58".6 and $19^{\prime \prime} .6$, and the angular resolutions achieved were $\left(4{ }^{\prime \prime} 9 \times 44^{\prime \prime} .5\right)$ and $\left(00^{\prime \prime} 61 \times 00^{\prime \prime} .55\right)$ with position angles (PA) of $-80^{\circ}$ and $+34^{\circ}$ for the $3 \mathrm{~mm}$ and $1 \mathrm{~mm}$ transitions, respectively. At the adopted distance of Mrk 273, these resolutions correspond to $(3.7 \times 3.4) \mathrm{kpc}$ and $(460 \times 420) \mathrm{pc}$ spatial scales.

Given the NOEMA configurations used, the maximum recoverable scales for our $1 \mathrm{~mm}$ and $3 \mathrm{~mm}$ observations are 2.5 and 17" respectively, which are well above (about one order of magnitude) the emission sizes of the molecules (see Sect. 3.3.1 and Table 3). Furthermore, our $\mathrm{HCN}$ and $\mathrm{HCO}^{+}$ fluxes are consistent with those obtained by Graciá-Carpio et al. (2008) with the IRAM 30 m single-dish telescope. We can, therefore, safely claim that there was no flux filtered out in our observations.

\subsection{Herschel}

The $\mathrm{H}_{2} \mathrm{O}$ data were taken with the Photodetector Array Camera and Spectrometer (PACS; Ott 2010; Poglitsch et al. 2010) and the Spectral and Photometric Imaging Receiver (SPIRE; Griffin et al. 2010) on December 16, 2012 and November 21, 2010 respectively. The absorption water lines observed with Herschel/PACS are new detections in Mrk 273, while the emission transitions detected with Herschel/SPIRE were already reported by Lu et al. (2017). The PACS observations ${ }^{3}$ (PI: González-Alfonso) were performed in high spectral sampling

\footnotetext{
http://www . iram. fr/IRAMFR/GILDAS

OBSIDs:1342257290-1342257294.
} 
range spectroscopy mode in first and second orders of the grating, resulting in a velocity resolution of $\sim 170-265 \mathrm{~km} \mathrm{~s}^{-1}$. The spectra were reduced with the standard product generation pipeline version 14.2.0. The nuclear FIR emission from Mrk 273 was unresolved with the PACS 5 spaxel $\times 5$ spaxel IFU with 9 '.4 $\times 9$ '.4 spaxels, so the spectra were extracted using the point source correction task available in the Herschel interactive processing environment (HIPE; Ott 2010) version 14.0.1. The spectra were then scaled to the integrated flux level of the central $3 \times 3$ PACS spaxels to compensate for pointing offsets and jitter which act to move flux out of the central spaxel. The $\mathrm{H}_{2} \mathrm{O}$ spectra were sampled in velocities of $20-40 \mathrm{~km} \mathrm{~s}^{-1}$ per channel width. Polynomial baselines of orders lower than three were then removed, and the final root mean squares (rms) are $0.2-0.3 \mathrm{Jy} \mathrm{km} \mathrm{s}^{-1}$. The lines were fitted with Gaussian line profiles using the GILDAS software CLASS (Fig. 3).

The SPIRE observation ${ }^{4}$ (PI: P. P. van der Werf) was conducted using a single pointing centred on Mrk 273 in high-spectral-resolution sparse image sampling mode with a resolution of $1.2 \mathrm{GHz}\left(\sim 250-360 \mathrm{~km} \mathrm{~s}^{-1}\right)$ in the two observing bands $(447-989 \mathrm{GHz}$ and $958-1545 \mathrm{GHz})$. In total, 99 repetitions (198 FTS scans) were performed for a total on source time of $13187 \mathrm{~s}$. The data reduction was done with the standard single pointing pipeline available in HIPE version 14.0.1 and a bootstrap method was used to extract the line fluxes. The individual scans were averaged together and a polynomial baseline was extracted from each detector before all lines were fitted simultaneously using Gaussian profiles convolved with a sinc function (Fig. 4). After 1000 repetitions of this procedure, Gaussians were fitted to the resulting flux distribution of each line to get the mean line flux together with its standard deviation.

\section{Results}

\subsection{Continuum}

We first created the $3 \mathrm{~mm}(\sim 89 \mathrm{GHz})$ continuum visibilities including only the channels free of line emission after smoothing to the final spectral resolution. These visibilities were then subtracted from the total emission in the uv plane. Using the task $u v \_f i$ within the MAPPING package, we measured the size and flux of the $3 \mathrm{~mm}$ continuum. Our values were calculated by fitting an elliptical Gaussian in the Fourier plane. Circular and elliptical fits gave consistent values within the errors. Given that the images of the continuum and line emissions are quite round, but not entirely, we opted for using elliptical fits in order to take into account small asymmetries in the emission. We measured a spatially integrated flux density of the $3 \mathrm{~mm}$ continuum of $8.24 \pm 0.07 \mathrm{mJy}$ and a deconvolved size of $(1.92 \pm 0.06)^{\prime \prime} \times(1.83 \pm 0.07)^{\prime \prime}$ with a PA $=(-90 \pm 30)^{\circ}$. This is similar to the $111 \mathrm{GHz}$ continuum flux density of $11 \pm 2 \mathrm{mJy}$ obtained by Downes \& Solomon (1998).

Due to the very broad line widths at zero intensity $\left(500-1100 \mathrm{~km} \mathrm{~s}^{-1}\right)$ in the $1 \mathrm{~mm}$ band $(\sim 265 \mathrm{GHz})$ and the narrow bandwidth of the correlator, almost all channels contain line emission. Therefore, the continuum visibilities were created using only nine line-emission-free channels (15\% of the total number of channels) at both edges of the spectrum. Including more channels could potentially lead to an overestimation of the continuum flux. A fit of an elliptical Gaussian in the uv plane gives an integrated flux of $28.6 \pm 0.9 \mathrm{mJy}$, with a deconvolved size (FWHM - full with at half maximum - of the Gaussian) of

\footnotetext{
4 OBSID:1342209850.
}

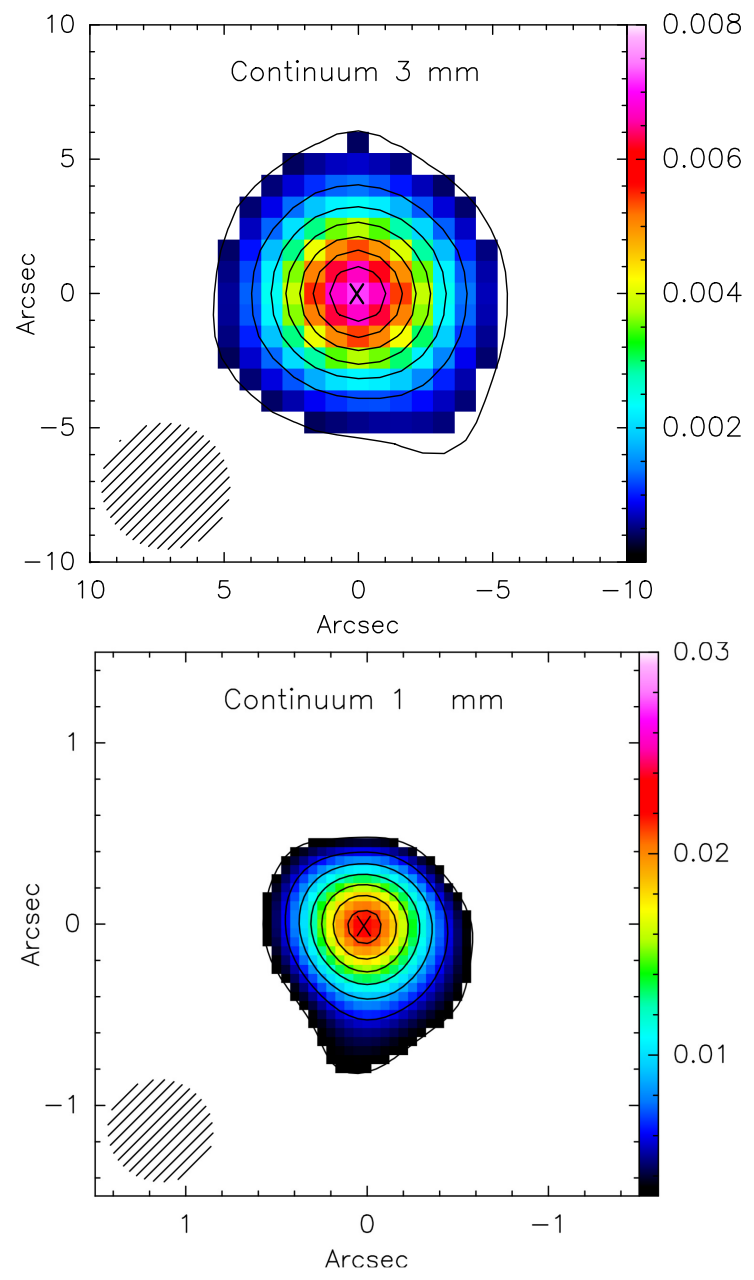

Fig. 1. Continuum maps at $3 \mathrm{~mm}$ and $1 \mathrm{~mm}$. Contour levels start at a significance of $5 \sigma$ with respect to the rms measured in both images $\left(\mathrm{rms}=0.07 \mathrm{mJy} \mathrm{channel}^{-1}\right.$ and $0.6 \mathrm{mJy} \mathrm{channel}^{-1}$ for the $3 \mathrm{~mm}$ and $1 \mathrm{~mm}$ maps respectively). The contour steps are 1 and $3 \mathrm{mJy} \mathrm{km} \mathrm{s}^{-1}$ beam $^{-1}$. The crosses at the centres mark the continuum peaks, which we take as the location of the northern nuclear source (see Sect. 1). The synthesised beams are shown in the bottom-left corner. The colour flux scales are in $\mathrm{Jy} \mathrm{km} \mathrm{s}^{-1}$ beam ${ }^{-1}$.

$(0.36 \pm 0.03)^{\prime \prime} \times(0.27 \pm 0.03)^{\prime \prime} \sim(270 \times 200 \mathrm{pc})$ with a position angle of $(24 \pm 12)^{\circ}$. The integrated intensities of the continuum at $1 \mathrm{~mm}$ and $3 \mathrm{~mm}$ are plotted in Fig. 1 .

\subsection{Line profiles}

\subsubsection{Nuclear emission}

Figure 2 shows the spectra of all observed lines with NOEMA extracted from the whole region (top panels), and from the central pixel of the observations (bottom panels). The total emission was integrated using masks in the moment zero maps of the $\mathrm{HCO}^{+}(1-0)$ and $(3-2)$ lines, which show the most extended emission at $1 \mathrm{~mm}$ and $3 \mathrm{~mm}$ (Table 3 ). These masks were also used to integrate the emission of the more compact species observed at similar frequencies (i.e. one mask over $\mathrm{HCO}^{+}(1-0)$ for all $3 \mathrm{~mm}$ species, and other mask over $\mathrm{HCO}^{+}(3-2)$ for all $1 \mathrm{~mm}$ species). While $\mathrm{HOC}^{+}(1-0)$ is not detected, the $(3-2)$ transition is seen arising from a very compact region near the nucleus. When integrating the overall flux of the map, the emission of the $\mathrm{HOC}^{+}(3-2)$ line drops below the noise and, therefore, 

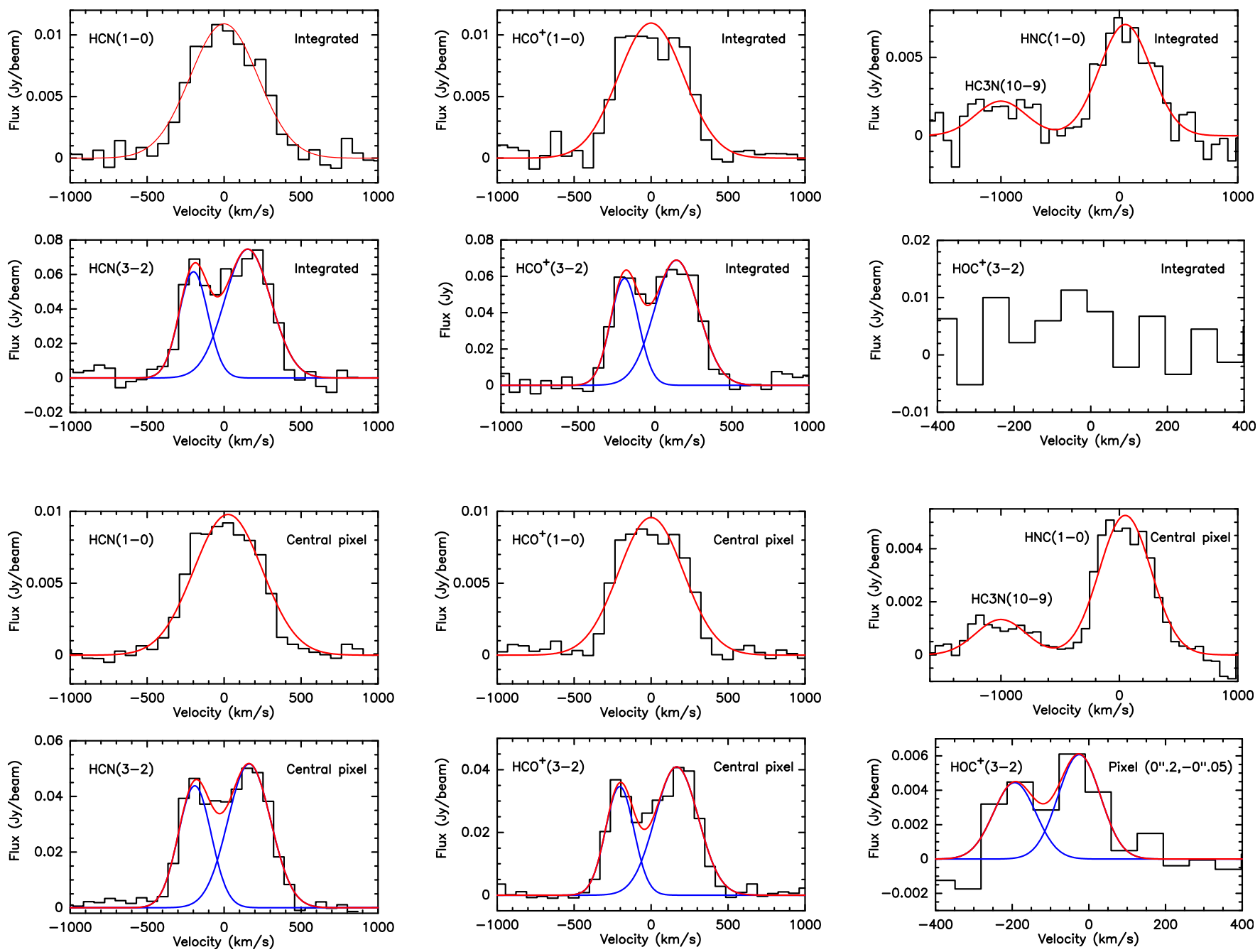

Fig. 2. Emission lines detected with NOEMA (black histograms) and Gaussian fits (total: red lines; if two components are present, individual components are displayed in blue colour). The velocity resolution is $68 \mathrm{~km} \mathrm{~s}^{-1}$ in all cases. The labels in the top right corners indicate if the spectra were extracted from the integrated emission ("Integrated", top panels), from the central pixel (bottom panels), or in the case of $\mathrm{HOC}^{+}$, from the pixel at $\left(0^{\prime \prime} 2,-0, \cdot 05\right)$.

for comparison with the other molecules, we measure its flux in the pixel where it peaks (see Sect. 3.3 for details). There, $\mathrm{HOC}^{+}(3-2)$ is detected with a signal-to-noise ratio $(\mathrm{S} / \mathrm{N})$ of seven. Additionally, its central velocity is blue-shifted compared to the other lines observed with NOEMA (Table 1).

The $3 \mathrm{~mm}$ lines $\left(\mathrm{HCN}, \mathrm{HCO}^{+}, \mathrm{HNC}\right.$ and $\mathrm{HOC}^{+}(1-0)$, and $\mathrm{HC}_{3} \mathrm{~N}(10-9)$ ) have roughly Gaussian-like profiles, although their peaks are slightly flat-topped. These lines were fitted with single Gaussian velocity profiles (Fig. 2). On the other hand, the $1 \mathrm{~mm}$ lines $\left(\mathrm{HCN}, \mathrm{HCO}^{+}\right.$and $\left.\mathrm{HOC}^{+}(3-2)\right)$ are double-peaked. To account for these profiles, we fitted two Gaussians (their parameters are listed in Table 1). The intensity of the dip between the double peaks is $19 \mathrm{mJy}$ and $26 \mathrm{mJy}$ for the $\mathrm{HCN}$ and $\mathrm{HCO}^{+}(3-2)$ lines, respectively. The dip appears at slightly blueshifted velocities, specifically at $-30 \mathrm{~km} \mathrm{~s}^{-1}$ for $\operatorname{HCO}^{+}(3-2)$, and $-60 \mathrm{~km} \mathrm{~s}^{-1}$ for $\mathrm{HCN}(3-2)$. The nature of these doublepeaked profiles is further discussed in Sect. 4.1.

Figures 3 and 4 show the $\mathrm{H}_{2} \mathrm{O}$ line profiles observed with Herschel PACS and SPIRE and the best Gaussians fits. All transitions were fitted by single Gaussian profiles (for the SPIRE lines, the Gaussians were convolved with a sinc function, Sect. 2.2). $\mathrm{H}_{2} \mathrm{O}\left(5_{24}-4_{13}\right)$ is partially blended with an $\mathrm{OH}$ line at $71 \mu \mathrm{m}$. To disentangle the emission of the two species, we fitted a double Gaussian profile to the observed spectrum.

It is important to note that all Gaussian fits to the water lines observed with PACS are blue-shifted to velocities between $-20 \mathrm{~km} \mathrm{~s}^{-1}$ and $-140 \mathrm{~km} \mathrm{~s}^{-1}$ (Table 2). Interestingly, these values are, to within the errors, the same as the velocities of the dips in the profiles of $\mathrm{HCO}^{+}$and $\mathrm{HCN}(3-2)$. The connection between the two is discussed in Sect. 4.1.

\subsubsection{High-velocity emission}

None of the $3 \mathrm{~mm}$ and FIR water lines show obvious extended line wings, which would reveal the Mrk 273 molecular outflow previously detected with $\mathrm{CO}, \mathrm{OH}$, and $\mathrm{H}_{2}$ by U et al. (2013), Veilleux et al. (2013), Cicone et al. (2014), and González-Alfonso et al. (2017). For the $1 \mathrm{~mm}$ observations, the spectrum of the overall integrated emission has no evident signatures of line wings either. However, in the central pixel, the HCN profile exhibits a line wing that extends between -400 and $\sim 1000 \mathrm{~km} \mathrm{~s}^{-1}$, while the red side shows no wing (Fig. 5). This emission is detected with an $\mathrm{S} / \mathrm{N}$ of $\sim 5$.

We used the JPL catalog (Pickett et al. 1998) to look for lines arising from 266.2 to $266.9 \mathrm{GHz}$, which correspond to 
Table 1. Gaussian fit parameters to the emission lines detected with NOEMA.

\begin{tabular}{|c|c|c|c|c|c|}
\hline & $\begin{array}{c}\text { Flux } \\
\left(\mathrm{Jy} \mathrm{km} \mathrm{s}^{-1}\right)^{a}\end{array}$ & $\begin{array}{l}\text { Line peak } \\
(\mathrm{mJy})^{a}\end{array}$ & $\begin{array}{l}\text { FWHM } \\
\left(\mathrm{km} \mathrm{s}^{-1}\right)\end{array}$ & $\begin{array}{c}\text { Central velocity } \\
\left(\mathrm{km} \mathrm{s}^{-1}\right)\end{array}$ & Pixel \\
\hline $\operatorname{HCN}(1-0)$ & $6.0 \pm 0.9$ & 10.9 & $520 \pm 85$ & $0 \pm 0$ & All \\
\hline $\mathrm{HCO}^{+}(1-0)$ & $5.8 \pm 0.8$ & 11.0 & $501 \pm 78$ & $0 \pm 0$ & All \\
\hline $\mathrm{HNC}(1-0)$ & $3.9 \pm 0.4$ & 7.1 & $517 \pm 59$ & $50 \pm 0$ & All \\
\hline $\mathrm{HC}_{3} \mathrm{~N}(10-9)$ & $1.2 \pm 0.3$ & 2.2 & $500 \pm 0$ & $0 \pm 0$ & All \\
\hline $\mathrm{HCN}(1-0)$ & $5.4 \pm 0.8$ & 9.8 & $524 \pm 79$ & $26 \pm 0$ & $\left(0^{\prime \prime}, 0^{\prime \prime}\right)$ \\
\hline $\mathrm{HCO}^{+}(1-0)$ & $5.0 \pm 0.7$ & 9.6 & $495 \pm 78$ & $0 \pm 0$ & $\left(0^{\prime \prime}, 0^{\prime \prime}\right)$ \\
\hline $\mathrm{HNC}(1-0)$ & $2.9 \pm 0.3$ & 5.2 & $516 \pm 51$ & $50 \pm 0$ & $\left(0^{\prime \prime}, 0^{\prime \prime}\right)$ \\
\hline $\mathrm{HC}_{3} \mathrm{~N}(10-9)$ & $0.7 \pm 0.2$ & 1.3 & $500 \pm 0$ & $0 \pm 0$ & $\left(0^{\prime \prime}, 0^{\prime \prime}\right)$ \\
\hline $\operatorname{HCN}(3-2)_{1}$ & $14.9 \pm 0.8$ & 63.3 & $220 \pm 179$ & $-198 \pm 71$ & All \\
\hline $\operatorname{HCN}(3-2)_{2}$ & $28.8 \pm 0.5$ & 76.6 & $354 \pm 170$ & $154 \pm 83$ & All \\
\hline $\mathrm{HCO}^{+}(3-2)_{1}$ & $13.3 \pm 0.7$ & 58.7 & $213 \pm 136$ & $-197 \pm 66$ & All \\
\hline $\mathrm{HCO}^{+}(3-2)_{2}$ & $24.7 \pm 4.2$ & 68.7 & $338 \pm 159$ & $140 \pm 75$ & All \\
\hline $\operatorname{HCN}(3-2)_{1}$ & $11.7 \pm 0.6$ & 43.8 & $251 \pm 130$ & $-190 \pm 63$ & $\left(0^{\prime \prime}, 0^{\prime \prime}\right)$ \\
\hline $\operatorname{HCN}(3-2)_{2}$ & $18.0 \pm 0.1$ & 51.7 & $328 \pm 134$ & $164 \pm 63$ & $\left(0^{\prime \prime}, 0^{\prime \prime}\right)$ \\
\hline $\mathrm{HCO}^{+}(3-2)_{1}$ & $8.0 \pm 1.0$ & 34.6 & $216 \pm 0$ & $-203 \pm 92$ & $\left(0^{\prime \prime}, 0^{\prime \prime}\right)$ \\
\hline $\mathrm{HCO}^{+}(3-2)_{2}$ & $14.4 \pm 0.6$ & 40.9 & $331 \pm 199$ & $166 \pm 89$ & $\left(0^{\prime \prime}, 0^{\prime \prime}\right)$ \\
\hline $\mathrm{HOC}^{+}(3-2)_{1}$ & $0.6 \pm 0.3$ & 4.4 & $130 \pm 0$ & $-197 \pm 0$ & $\left(0.2^{\prime \prime},-0.05^{\prime \prime}\right)$ \\
\hline $\mathrm{HOC}^{+}(3-2)_{2}$ & $1.0 \pm 0.2$ & 7.6 & $130 \pm 0$ & $-27 \pm 0$ & $\left(0.2^{\prime \prime},-0.05^{\prime \prime}\right)$ \\
\hline
\end{tabular}

Notes. The last column indicates whether the spectra were extracted from only one pixel, or from all pixels showing significant emission. ${ }^{(a)}$ The units of flux and line peak estimated in a single pixel are $\mathrm{Jy} \mathrm{km} \mathrm{s}^{-1}$ beam $^{-1}$ and mJy beam ${ }^{-1}$, respectively. For the $\left(J-J^{\prime}\right)=(3-2)$ lines near $\lambda=1 \mathrm{~mm}$, we denote the two Gaussian components with the subscripts "1", and " 2 ". Parameters with zero errors were fixed.
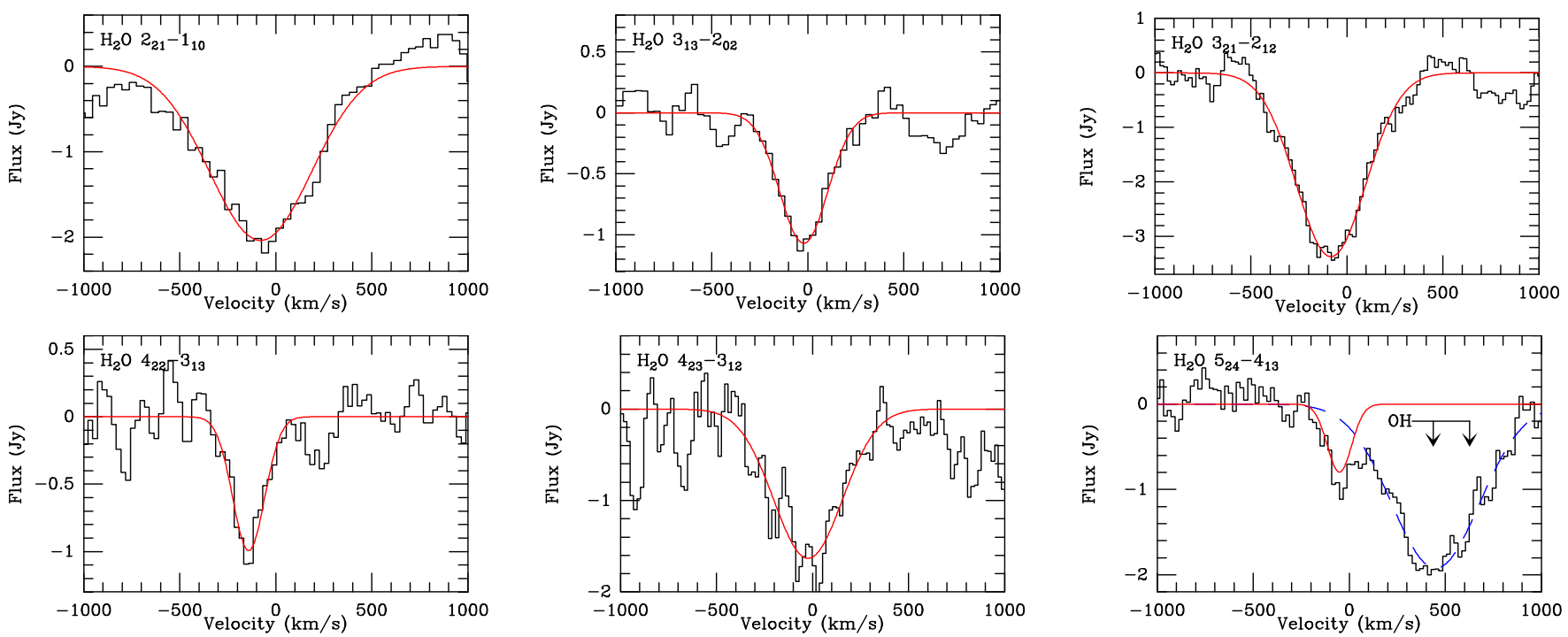

Fig. 3. $\mathrm{H}_{2} \mathrm{O}$ absorption lines (black histograms) detected with Herschel/PACS and Gaussian fits (red lines). The velocity sampling is $20-40 \mathrm{~km} \mathrm{~s}^{-1}$.

the velocity range $[-350,-1200] \mathrm{km} \mathrm{s}^{-1}$ where the $\mathrm{HCN}(3-2)$ wing-like feature is seen. $\mathrm{CH}_{2} \mathrm{NH}\left(4_{1,3}-3_{1,2}\right)$ is the most likely line arising at these frequencies, with an energy level of $E_{\text {low }}=19 \mathrm{~K}$. This transition was detected in the LIRGs IC 860 and $\mathrm{Zw}$ 49-57, where its flux density is three to four times fainter than $\mathrm{HCN}(3-2)$ (Aalto et al. 2015b). Assuming a similar ratio and excitation conditions in Mrk 273, $\mathrm{CH}_{2} \mathrm{NH}$ would then have a peak flux between 4.5 and $6 \mathrm{mJy}$, that is, brighter than the emission we see. The HCN shoulder does not have a (single or double) Gaussian profile similar to the detected lines, but has the shape of a line wing. For these reasons, it seems unlikely that the emission comes from the $\mathrm{CH}_{2} \mathrm{NH}$ line, although a potential contamination cannot be ruled out.
The two nuclei of Arp 220 have $\operatorname{HCN}(3-2)$ and $(4-3)$ blue-shifted wings very similar to what we observe here in Mrk 273 (Martín et al. 2016). The fact that these bumps appear in both $\mathrm{HCN}$ transitions, while there is no corresponding $\mathrm{CH}_{2} \mathrm{NH}$ line close to the $\mathrm{HCN}(4-3)$ frequency, strengthens our claim that the line wing in Mrk 273 comes from $\operatorname{HCN}(3-2)$, and that it is tracing gas outflowing at high velocities. In summary, given the feature intensity, spectral shape, and integrated emission (see following section), we conclude that the $\operatorname{HCN}(3-2)$ blue-shifted shoulder comes from the outflowing gas moving at approximate velocities between -400 and $-1000 \mathrm{~km} \mathrm{~s}^{-1}$. In Sects. 3.3.2 and 4.2 we discuss in more detail the properties of this molecular outflow. 

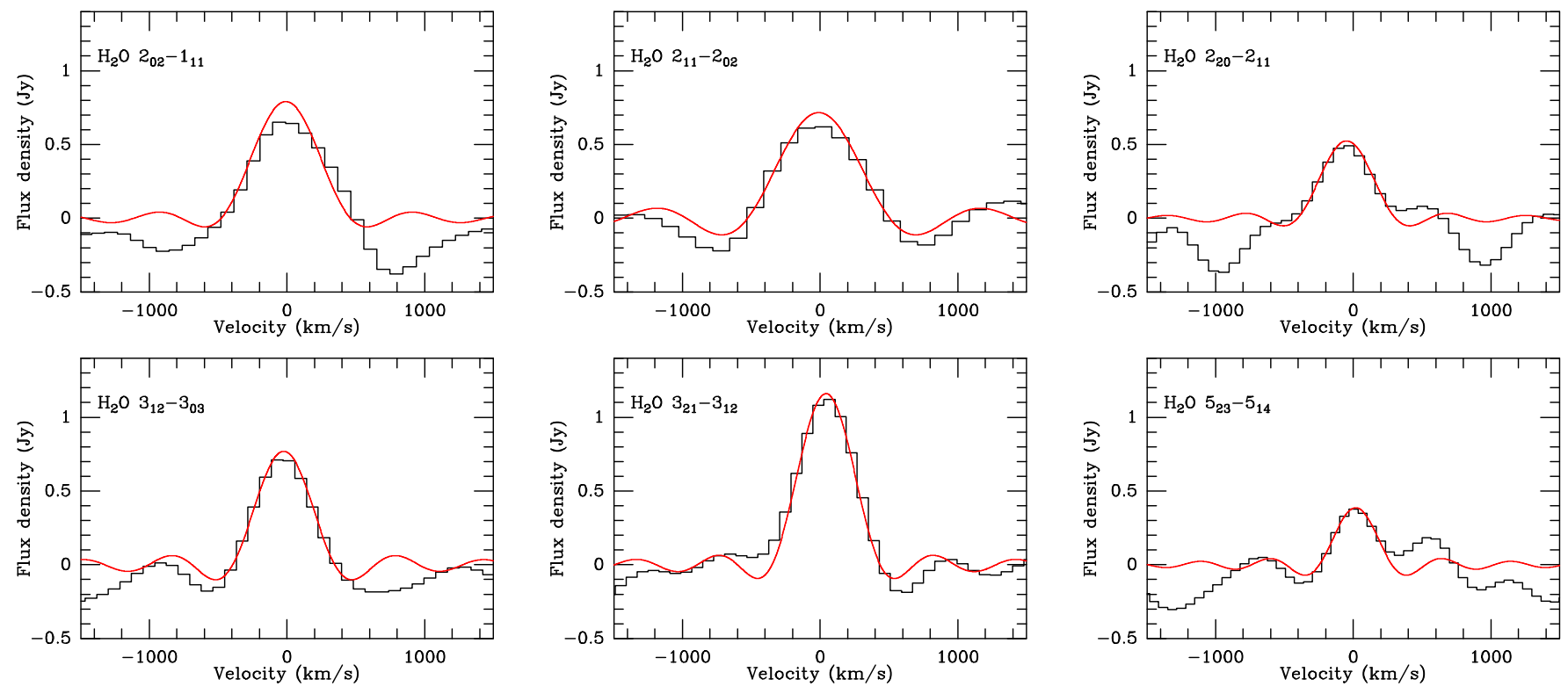

Fig. 4. $\mathrm{H}_{2} \mathrm{O}$ lines (black histograms) detected with Herschel/SPIRE, and Gaussians fits (convolved with sinc functions).

Table 2. Gaussian fit parameters to the water lines detected with Herschel.

\begin{tabular}{lcccccc}
\hline \hline & $\begin{array}{c}\lambda_{\text {rest }} \\
(\mu \mathrm{m})\end{array}$ & $\begin{array}{c}E_{\text {low }} / E_{\text {upper }}^{a} \\
(\mathrm{~K})\end{array}$ & $\begin{array}{c}\text { Flux } \\
\left(\mathrm{Jy} \mathrm{km} \mathrm{s}^{-1}\right)\end{array}$ & $\begin{array}{c}\text { Line peak } \\
(\mathrm{mJy})\end{array}$ & $\begin{array}{c}\text { FWHM } \\
\left(\mathrm{km} \mathrm{s}^{-1}\right)\end{array}$ & $\begin{array}{c}\text { Central velocity } \\
\left(\mathrm{km} \mathrm{s}^{-1}\right)\end{array}$ \\
\hline $\mathrm{H}_{2} \mathrm{O}\left(2_{2,1}-1_{1,0}\right)$ & 108.073 & 61 & $-1356 \pm 87$ & $-2.0 \pm 0.1$ & $625 \pm 48$ & $-79 \pm 21$ \\
$\mathrm{H}_{2} \mathrm{O}\left(3_{1,3}-2_{0,2}\right)$ & 138.528 & 101 & $-334 \pm 46$ & $-1.1 \pm 0.1$ & $294 \pm 49$ & $-21 \pm 20$ \\
$\mathrm{H}_{2} \mathrm{O}\left(3_{2,1}-2_{1,2}\right)$ & 75.381 & 114 & $-1531 \pm 60$ & $-3.4 \pm 0.2$ & $427 \pm 19$ & $-82 \pm 8$ \\
$\mathrm{H}_{2} \mathrm{O}\left(4_{2,2}-3_{1,3}\right)$ & 57.636 & 205 & $-240 \pm 62$ & $-1.0 \pm 0.1$ & $215 \pm 70$ & $-140 \pm 26$ \\
$\mathrm{H}_{2} \mathrm{O}\left(4_{2,3}-3_{1,2}\right)$ & 78.742 & 275 & $-733 \pm 138$ & $-1.6 \pm 0.2$ & $422 \pm 94$ & $-23 \pm 38$ \\
$\mathrm{H}_{2} \mathrm{O}\left(5_{2,4}-4_{1,3}\right)$ & 71.067 & 396 & $-127 \pm 28$ & $-0.8 \pm 0.1$ & $150 \pm 35$ & $-51 \pm 14$ \\
$\mathrm{H}_{2} \mathrm{O}\left(2_{0,2}-1_{1,1}\right)$ & 303.456 & 101 & $421 \pm 74$ & $0.8 \pm 0.1$ & $207 \pm 41$ & $-24 \pm 75^{b}$ \\
$\mathrm{H}_{2} \mathrm{O}\left(2_{1,1}-2_{0,2}\right)$ & 398.643 & 137 & $408 \pm 42$ & $0.71 \pm 0.06$ & $164 \pm 40$ & $-26 \pm 32^{b}$ \\
$\mathrm{H}_{2} \mathrm{O}\left(2_{2,0}-2_{1,1}\right)$ & 243.974 & 196 & $220 \pm 67$ & $0.5 \pm 0.1$ & $148 \pm 75$ & $-63 \pm 56^{b}$ \\
$\mathrm{H}_{2} \mathrm{O}\left(3_{1,2}-3_{0,3}\right)$ & 273.193 & 249 & $223 \pm 52$ & $0.8 \pm 0.1$ & $137 \pm 38$ & $-39 \pm 38^{b}$ \\
$\mathrm{H}_{2} \mathrm{O}\left(3_{2,1}-3_{1,2}\right)$ & 257.795 & 305 & $545 \pm 62$ & $1.2 \pm 0.1$ & $171 \pm 3$ & $28 \pm 25^{b}$ \\
$\mathrm{H}_{2} \mathrm{O}\left(5_{2,3}-5_{1,4}\right)$ & 212.526 & 642 & $128 \pm 43$ & $0.4 \pm 0.1$ & $65 \pm 51$ & $3 \pm 54^{b}$ \\
\hline
\end{tabular}

Notes. Some lines are affected by strong noise that increases the errors in the Gaussian fittings. ${ }^{(a)}$ The first six $\mathrm{H}_{2} \mathrm{O}$ lines were detected in absorption with PACS, and we show the energy of their lower level. The last six lines were detected with SPIRE in emission, so we show the energy of their upper level. ${ }^{(b)}$ Due to the low velocity resolution of the SPIRE instrument $\sim 250-360 \mathrm{~km} \mathrm{~s}^{-1}$, these values are very uncertain and we do not consider them in our discussion.

\subsection{Moment maps of $\mathrm{HCN}, \mathrm{HCO}^{+}, \mathrm{HNC}$ and $\mathrm{HOC}^{+}$}

\subsubsection{Position and size of the nuclear emission}

Figure 6 and Table 1 show the integrated intensities of the HCN, $\mathrm{HCO}^{+}, \mathrm{HNC}$ and $\mathrm{HOC}^{+}$lines. The deconvolved emission sizes and position angles of all lines were measured in the uv plane by fitting elliptical Gaussians with the task $u v_{-}$fit within MAPPING, and are listed in Table 3 . The continuum and the $\mathrm{HCN}, \mathrm{HCO}^{+}$, and $\mathrm{HNC}$ lines have their peak intensities at the central pixel, which we take as the location of the northern nuclear source. On the contrary, the $\mathrm{HOC}^{+}$maximum is found at an offset $\left(0 \prime .2,-00^{\prime \prime} 05\right)$, corresponding to $(152,-38) \mathrm{pc}$ to the south-east. The position accuracy of our observations can be calculated from

$\Delta \alpha, \Delta \delta \simeq 0.6 \times(S / N)^{-1} \times \theta_{\mathrm{b}}$,

where $\Delta \alpha, \Delta \delta$ are the errors in RA and Dec, and $\theta_{\mathrm{b}}$ is the synthesised beam size (Condon 1997; Ivison et al. 2007). For
$\mathrm{HOC}^{+}(3-2)$, we have a $\Delta \alpha, \Delta \delta \simeq 0^{\prime \prime} .1(\sim 80 \mathrm{pc})$, confirming our claim that its shift is real, at least in RA (because the $\mathrm{HOC}^{+}$offset in declination is smaller than our position accuracy). In Sect. 4.6, we further discuss the origin of $\mathrm{HOC}^{+}$.

Downes \& Solomon (1998) estimated a CO (1 - 0) emission deconvolved size of $\left(0^{\prime \prime} .9 \times 00^{\prime \prime} 6\right)$ with a beam of $\left(1{ }^{\prime \prime} 4 \times 11^{\prime \prime} 3\right)$. We obtain larger sizes for the $\mathrm{HCN}$ and $\mathrm{HCO}^{+}(1-0)$ lines $\left(\sim\left(2^{\prime \prime} \times 2^{\prime \prime}\right)\right.$ equivalent to $\sim 1.5 \mathrm{kpc}$, Table 3$)$, most probably because we collect more emission within our larger beam of $\left(4^{\prime \prime} .9 \times 4^{\prime \prime} .5\right)$. In the following, we refer to this region as the outer disc. The $\mathrm{HCN}$ and $\mathrm{HCO}^{+}(3-2)$ lines, however, are confined to a much smaller region of $\left(0^{\prime \prime} .4 \times 00^{\prime \prime} .3\right)(\sim(300 \times 230)$ pc, Table 3$)$, implying that the denser, star-forming gas is more concentrated in the nucleus. We refer to this as the inner disc. Despite our high angular resolution at $267 \mathrm{GHz}$, the nucleus is still unresolved and does not show any structures in the moment zero maps, implying a very compact core that does not expand more than 


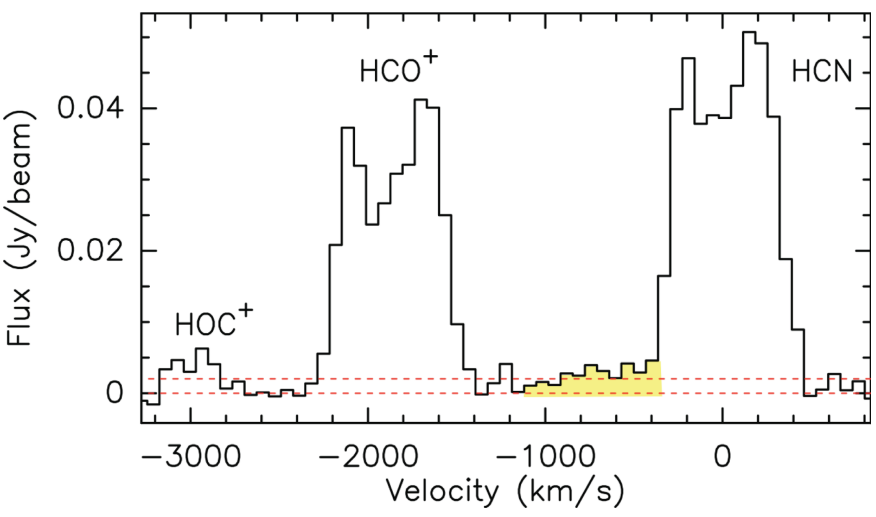

Fig. 5. Spectrum of the central pixel for the $\left(J-J^{\prime}\right)=(3-2)$ lines of $\mathrm{HCN}, \mathrm{HCO}^{+}$and $\mathrm{HOC}^{+}$. The baseline of order 0 and the $3 \times$ rms flux (calculated at the final velocity resolution of $68 \mathrm{~km} \mathrm{~s}^{-1}$ ) are marked with horizontal dashed lines. The outflow emission at the blue-shifted velocities of $\mathrm{HCN}$ is highlighted in yellow.

$<0$ '.3 (230 pc). Indeed, from our $\mathrm{H}_{2} \mathrm{O}$ modelling, we estimate the size of the core to be $\sim 50 \mathrm{pc}$ (see Sect. 3.4 for details).

$\mathrm{HC}_{3} \mathrm{~N}(10-9)$ and $\mathrm{HOC}^{+}(3-2)$ are faint (although we detect the latter with an $\mathrm{S} / \mathrm{N}$ of $\sim 7$ in the pixel where it peaks) and unresolved at our resolution. Therefore, we cannot measure their emission sizes.

\subsubsection{Integrated intensity of the outflow}

The moment-zero map of the outflow traced by $\mathrm{HCN}(3-2)$ (highlighted in yellow in Fig. 5) is shown in Fig. 7. After centering the spectrum at the $\mathrm{HCN}(3-2)$ rest frequency, we integrated the emission of all pixels in the velocity range $[-1100$, $-400] \mathrm{km} \mathrm{s}^{-1}$. Its morphology is composed of two main peaks of emission; a stronger one with an elliptical shape around the centre, and a fainter and rounder feature at $\sim 0.8^{\prime \prime}(\sim 600 \mathrm{pc})$ to the north. In the central pixel, the peak flux has a $\mathrm{S} / \mathrm{N}$ of $\sim 5$ (measured at the final velocity resolution), while the integrated flux density measured in the moment-zero map is $2.4 \mathrm{Jy} \mathrm{km} \mathrm{s}^{-1}$.

We measured the size of the outflow by fitting two ellipses to the main peaks seen in the moment-zero map (see Fig. 7). We take the size of the outflow as the projected distance between the central pixel and the centre of the northern ellipse. We measure a size of $00^{\prime \prime} 61 \pm 00^{\prime \prime} 05$, equivalent to $464 \mathrm{pc}$, with a position angle of $10 \pm 3^{\circ}$. The direction of the flow heading to the north is consistent with the outflowing gas detected with $\mathrm{CO}(1-0)$ by Cicone et al. (2014).

\subsubsection{Velocity fields and position-velocity maps}

Figures 8 and 9 show the velocity fields and position-velocity $(p-v)$ maps of $\mathrm{HCN}, \mathrm{HCO}^{+}$and $\mathrm{HNC}$. The velocity fields reveal the rotation of the Mrk 273 nuclear disc (see Downes \& Solomon 1998 for a detailed study of the disc properties). There are significant differences in the morphologies of the iso-velocity contours of the various observed lines. $\mathrm{HCN}$ and $\mathrm{HCO}^{+}(1-0)$ trace rotating gas in the outer disc showing a south-east to north-west direction. However, the less extended gas traced by HNC $(1-0)$ shows a north-east to south-west rotation, similar to the gas in the inner disc traced by the $\operatorname{HCN}(3-2)$ and $\mathrm{HCO}^{+}(3-2)$ lines. The rotation in the central $<0^{\prime \prime} .5$ follows the velocity fields traced by the warm and compact gas traced by $\mathrm{H}_{2}$, Br $\gamma$ and [FeII] (Medling et al. 2014; U et al. 2013). This is consistent with the two kinematic systems of the disc already discovered by Downes \& Solomon (1998) using the CO (1-0) and $\mathrm{CO}(2-1)$ lines with beam sizes $\left(1^{\prime \prime} \cdot 4,1^{\prime \prime \prime} 3\right)$ and $\left(0^{\prime \prime} 6,0^{\prime \prime} 6\right)$. The agreement between the Downes \& Solomon (1998) velocity fields and ours, despite the difference in angular resolution, suggests that this effect is not due to the larger beam size of our $3 \mathrm{~mm}$ data.

Figure 9 shows the $p-v$ diagrams of the $\mathrm{HCN}$ and $\mathrm{HCO}^{+}$ lines along cuts through the centre and perpendicular to the axes of rotation as plotted with dashed lines in Fig. 8. The outer disc traced by the $(1-0)$ lines shows maximum velocities $\pm 300 \mathrm{~km} \mathrm{~s}^{-1}$, though most of the gas exhibits velocities within the smaller range of $\pm 150 \mathrm{~km} \mathrm{~s}^{-1}$. The $p-v$ diagrams of $\mathrm{HCN}$ and $\mathrm{HCO}^{+}(3-2)$ show that the gas in the inner disc rotates faster, reaching maximum velocities of $\pm 400 \mathrm{~km} \mathrm{~s}^{-1}$, although the average speed is $\pm 200 \mathrm{~km} \mathrm{~s}^{-1}$ at the edges of the disc, which are separated by $0 \prime \prime 2(\sim 150 \mathrm{pc})$.

The dynamical mass of the inner, starbursting disc, measured from the kinematics of the $(3-2)$ lines, is $(4-5) \times 10^{9} M_{\odot}$, as calculated from $R V_{\text {rot }}^{2} / G$. Here, $V_{\text {rot }}$ is the average rotational velocity corrected for the $45^{\circ}$ inclination of the disc (Downes \& Solomon 1998), $R$ is the size of the emission in pc (Table 3), and $G$ is the gravitational constant.

\subsubsection{Velocity dispersions}

The velocity dispersions of the NOEMA data were calculated as

$\sigma_{v}=\frac{F W H M}{2 \times \sqrt{2 \times \ln (2)}}$.

The moment- 2 maps of $\mathrm{HCN}, \mathrm{HCO}^{+}$and $\mathrm{HNC}$ are shown in Fig. 10. All lines reach similar maximum dispersions of $500 \mathrm{~km} \mathrm{~s}^{-1}$. We explored the Toomre (1964) stability criterion, $Q \geq 1$, for the inner, starbursting, gaseous disc to check its stability against gravitational perturbations, as

$Q=\frac{\sigma_{v} \times \kappa}{\pi \times G \times \Sigma}$

where $\kappa$ is the epicyclic frequency, $G$ is the gravitational constant, and $\Sigma$ is the surface density of the gas. The surface density in the inner $380 \mathrm{pc}$ of Mrk 273 is $37500 M_{\odot} / \mathrm{pc}^{2}$ (Yun \& Scoville 1995). We note that this value was calculated in a region slightly larger than the radius of the inner disc (of $\sim 300 \mathrm{pc}$ ), but it is still a good approximation if compared to the size of the outer disc $(1.5 \mathrm{kpc})$. For the epicyclic frequency, we assumed a Keplerian disc. Therefore, $\kappa=\omega, \omega$ being the angular velocity. For consistency, we calculated $\omega$ in the same radius of $380 \mathrm{pc}$, and used the average rotational velocities from our HCN and $\mathrm{HCO}^{+}(3-2)$ velocity maps $\left(200 \mathrm{~km} \mathrm{~s}^{-1}\right.$, Figs. 8 and 9$)$. We obtain $Q=0.5$, which indicates that the inner disc is unstable and clumpy/turbulent enough to form further self-gravitating condensations of gas.

\subsection{5. $\mathrm{HCN}$ and $\mathrm{HCO}^{+}$channel-velocity maps}

Figures 11-14 show the channel maps of $\mathrm{HCN}$ and $\mathrm{HCO}^{+}(1-0)$ and $(3-2)$ between -500 and $+500 \mathrm{~km} \mathrm{~s}^{-1}$ in steps of $50 \mathrm{~km} \mathrm{~s}^{-1}$, with a beginning intensity contour level of $5 \sigma$. The $(1-0)$ lines show emission away from the nucleus in all directions. In particular, the $\mathrm{HCN}(1-0)$ channel map reveals gas in the northern direction as far as $10^{\prime \prime}$ from the centre at negative velocities, as well as elongations towards the south at $\pm 300 \mathrm{~km} \mathrm{~s}^{-1}$. 

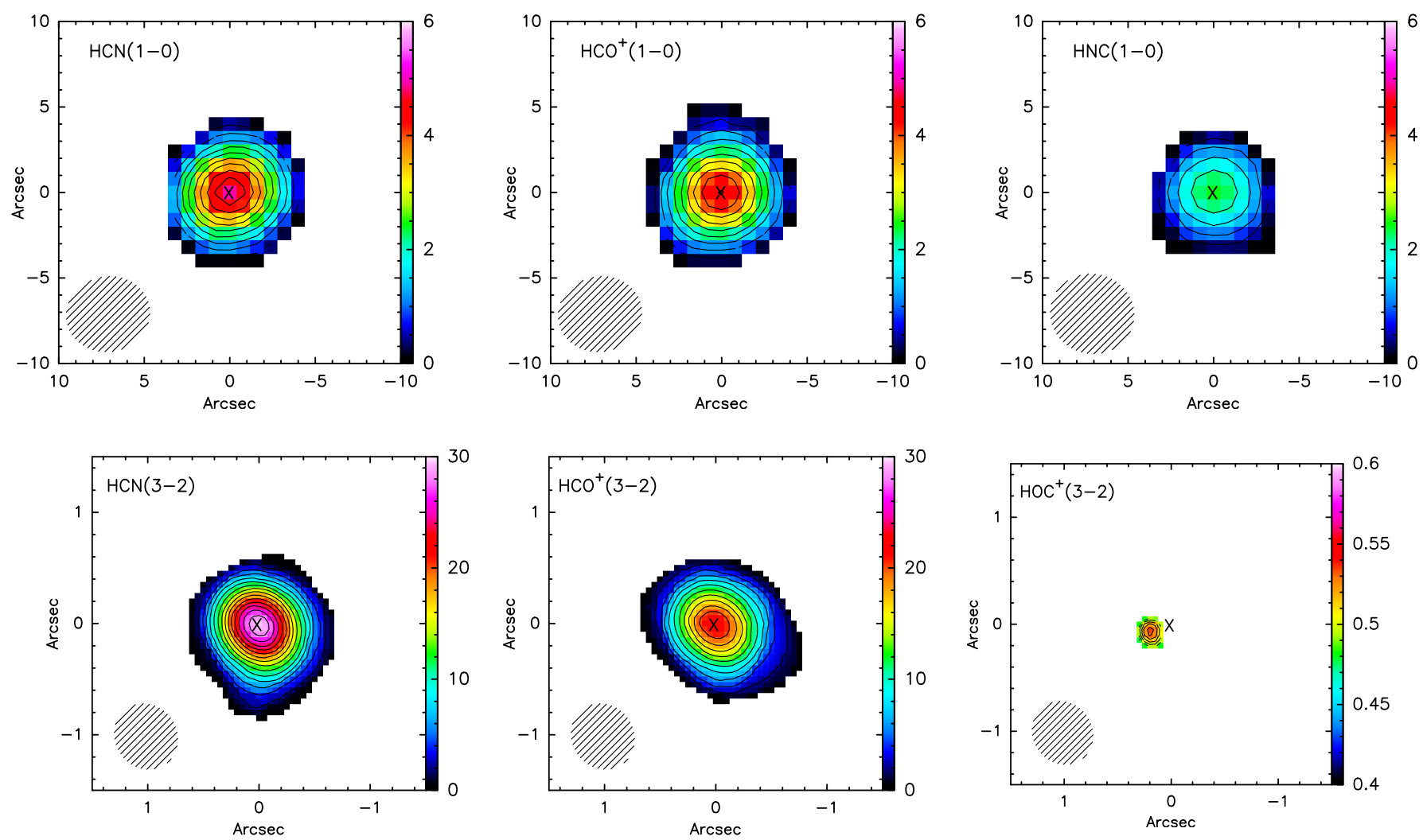

Fig. 6. Integrated intensities (moment zero maps). Contour levels for $\mathrm{HCN}, \mathrm{HCO}^{+}$and $\mathrm{HNC}(1-0)$ go from 0 to $6 \mathrm{Jy} \mathrm{kms}^{-1}$ beam ${ }^{-1}$ with a step of $0.5 \mathrm{Jy} \mathrm{kms}^{-1}$ beam $^{-1}$. For $\mathrm{HCN}(3-2)$ and $\mathrm{HCO}^{+}(3-2)$ the levels range from 2 to $30 \mathrm{Jy} \mathrm{kms}^{-1}$ beam ${ }^{-1}$ with a step of $2 \mathrm{Jy} \mathrm{kms}^{-1}$ beam ${ }^{-1}$. For $\mathrm{HOC}^{+}(3-2)$, the contours are from 0.4 to $0.6 \mathrm{Jy} \mathrm{kms}^{-1}$ beam $^{-1}$ with a step of $0.04 \mathrm{Jy} \mathrm{kms}^{-1}$ beam $^{-1}$. We highlight the different scales between the $(1-0)$ and the $(3-2)$ lines. The crosses in the centre of each panel indicate the location of the nuclear source. The synthesised beam is shown in the bottom-left corner of each panel. North is up, and east is to the left.

Table 3. Global properties of the lines detected with NOEMA.

\begin{tabular}{lccccc}
\hline \hline & $\begin{array}{c}v_{\text {rest }} \\
(\mathrm{MHz})\end{array}$ & $\mathrm{K} \mathrm{Jy}^{-1}$ & $\begin{array}{c}\mathrm{Size}^{a} \\
\left({ }^{\prime}\right)\end{array}$ & $\begin{array}{c}\mathrm{PA}^{a} \\
\left({ }^{\circ}\right)\end{array}$ & $\begin{array}{c}L^{\prime} \\
\left(\mathrm{K} \mathrm{km} \mathrm{s}^{-1} \mathrm{pc}^{2}\right)\end{array}$ \\
\hline $\mathrm{HCN}(1-0)$ & 88631.8 & 7.1675 & $(2.0 \pm 0.2) \times(1.6 \pm 0.2)$ & $-39 \pm 20$ & $2.5 \times 10^{8}$ \\
$\mathrm{HCO}^{+}(1-0)$ & 89188.6 & 7.0783 & $(2.2 \pm 0.2) \times(1.7 \pm 0.2)$ & $1 \pm 16$ & $2.8 \times 10^{8}$ \\
$\mathrm{HNC}(1-0)$ & 90663.6 & 6.4696 & $(2.4 \pm 0.4) \times(2.0 \pm 0.3)$ & $-36 \pm 35$ & $2.2 \times 10^{8}$ \\
$\mathrm{HC}{ }_{3}(10-9)$ & 90979.0 & 6.4248 & $<1.6$ & - & - \\
$\mathrm{HCN}(3-2)$ & 265886.4 & 51.4126 & $(0.35 \pm 0.01) \times(0.31 \pm 0.01)$ & $68 \pm 11$ & $4.4 \times 10^{8}$ \\
$\mathrm{HCO}^{+}(3-2)$ & 267557.6 & 50.7724 & $(0.40 \pm 0.01) \times(0.32 \pm 0.01)$ & $71 \pm 5$ & $2.6 \times 10^{8}$ \\
$\mathrm{HOC}^{+}(3-2)$ & 268451.1 & 50.4350 & $<0.3$ & - & - \\
\hline
\end{tabular}

Notes. ${ }^{(a)}$ Calculated as the FWHM of the elliptical Gaussian fitted to the line emission in the $u v$-plane (see Sect. 3.3.1). The line luminosity is $L^{\prime}=\pi R^{2} I$, where $R$ is the source size in column four in parsec $\left(1^{\prime \prime}=761 \mathrm{pc}\right)$, and $I$ is the integrated intensity (shown in Table 1$)$ in units of K km s ${ }^{-1}$.

Some extensions to the east and south-east are also seen in some channels. The $\mathrm{HCO}^{+}(1-0)$ emission is similarly extended, but the structure towards the south is perhaps the most distinct (e.g. channels $\left.\pm 150 \mathrm{~km} \mathrm{~s}^{-1}\right)$. Nevertheless, extensions to the north $\left( \pm 200 \mathrm{~km} \mathrm{~s}^{-1}\right.$ and $\left.\pm 300 \mathrm{~km} \mathrm{~s}^{-1}\right)$, east $\left( \pm 250 \mathrm{~km} \mathrm{~s}^{-1}\right)$, and west $\left(+150 \mathrm{~km} \mathrm{~s}^{-1}\right.$ and $\left.+250 \mathrm{~km} \mathrm{~s}^{-1}\right)$ are also seen.

The $\mathrm{HCN}$ and $\mathrm{HCO}^{+}(3-2)$ channel maps trace denser gas in the inner parts of the galactic disc $(\leq 300 \mathrm{pc})$, although some emission can be seen in the central $\pm 1^{\prime \prime}( \pm 800 \mathrm{pc})$, and their elongations are even more obvious than in the $(1-0)$ channel maps. HCN shows clear signs of emission towards the north in most channels (best seen between $-250 \mathrm{~km} \mathrm{~s}^{-1}$ and $+250 \mathrm{~km} \mathrm{~s}^{-1}$ ). On the other hand, the gas traced by $\mathrm{HCO}^{+}$ is more extended towards the south (e.g. $-200 \mathrm{~km} \mathrm{~s}^{-1}$ and $+50 \mathrm{~km} \mathrm{~s}^{-1}$ ).

The most significant extensions to the north (from the $\mathrm{HCN}$ maps) and to the south (from the $\mathrm{HCO}^{+}$maps) are signatures of outflowing gas, and are further discussed in Sect. 4.2.

\subsection{Modelling of $\mathrm{H}_{2} \mathrm{O}$}

We have used the library of $\mathrm{H}_{2} \mathrm{O}$ models generated by González-Alfonso et al. (2014) to fit the $\mathrm{H}_{2} \mathrm{O}$ emission and absorption observed in Mrk273. The models assume spherical symmetry, are non-local, and include excitation by both the FIR field emitted by warm dust (which is mixed with the $\mathrm{H}_{2} \mathrm{O}$ 


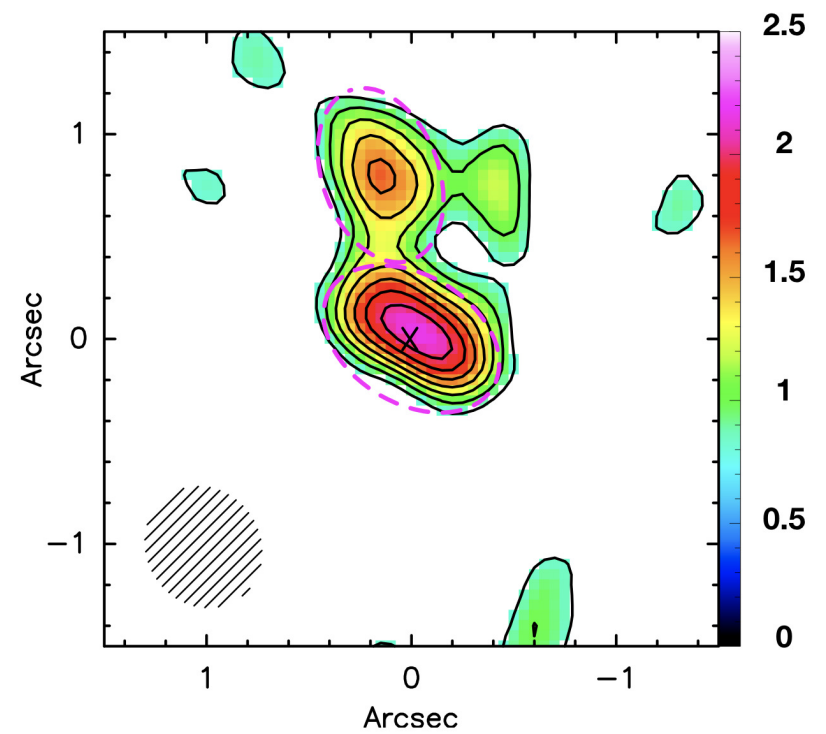

Fig. 7. Integrated intensity of the outflow seen in $\operatorname{HCN}(3-2)$ between $-1100 \mathrm{~km} \mathrm{~s}^{-1}$ and $-400 \mathrm{~km} \mathrm{~s}^{-1}$ (yellow region marked in Fig. 5). The cross in the centre marks the location of the nuclear source. The contours start with a $5 \sigma$ flux and go from $0.8 \mathrm{mJy} \mathrm{kms}^{-1}$ beam $^{-1}$ to $2.5 \mathrm{Jy} \mathrm{kms}^{-1}$ beam $^{-1}$ with steps of $0.2 \mathrm{mJy} \mathrm{kms}^{-1}$ beam $^{-1}$. The magenta dashed ellipses show the regions fitted to the two main components. The synthesised beam is plotted in the bottom-left corner.

molecules) and collisions with $\mathrm{H}_{2}$. The collisional rates were taken from Dubernet et al. (2009) and Daniel et al. (2011), and a gas-to-dust ratio of 100 was adopted (Wilson et al. 2008). The models assume uniform physical properties $\left(T_{\text {dust }}, T_{\text {gas }}\right.$, gas and dust densities, $\mathrm{H}_{2} \mathrm{O}$ abundance). The source is divided into a set of spherical shells where the statistical equilibrium level populations are calculated. We assume a $\mathrm{H}_{2} \mathrm{O}$ ortho-to-para ratio of three. Line broadening is simulated by including a microturbulent velocity $\left(V_{\text {turb }}\right)$, with no systemic motions.

The modelled line fluxes and continuum flux densities scale as $\left(R / D_{\mathrm{L}}\right)^{2}$ where $R$ is the source radius and $D_{\mathrm{L}}$ is the luminosity distance, so that they are easily scalable to the properties of any source. Following Falstad et al. (2017) and González-Alfonso et al. (2017), we have fit the line fluxes with a combination of $N_{C}$ model components by minimising $\chi^{2}$, with the radius $R$ of each component being the only free parameter that is varied. We required $N_{C}=2$ components to properly fit the PACS and SPIRE $\mathrm{H}_{2} \mathrm{O}$ fluxes simultaneously. Since the models also make specific predictions for the spectral energy distribution (SED) of each component, and since $\mathrm{H}_{2} \mathrm{O}$ probes the galaxy FIR emission responsible for its excitation and more specifically the transition from the mid- to the far-IR (González-Alfonso et al. 2010, 2014), we also included in the fit the continuum flux densities at 30 and $60 \mu \mathrm{m}$.

Figure 15 compares the observed $\mathrm{H}_{2} \mathrm{O}$ fluxes and SED with the results of our best model fit, and Table 4 lists the properties of the two model components (shown with blue and green colours in Fig. 15). The two components show very different behaviours relative to the $\mathrm{H}_{2} \mathrm{O}$ absorption and emission. We require a very compact (effective radius $R \sim 50 \mathrm{pc}$ ), very warm $\left(T_{\text {dust }} \sim 95 \mathrm{~K}\right)$, and very optically thick $\left(\tau_{100 \mu \mathrm{m}} \gtrsim 4\right)$ component (referred to as the "core") to account for the observed PACS absorption in several lines (primarily the $3_{13}-2_{02}$ line at $138 \mu \mathrm{m}$, the $4_{22}-3_{13}$ line at $58 \mu \mathrm{m}$, the $4_{23}-3_{12}$ line at $79 \mu \mathrm{m}$, and the $5_{24}-4_{13}$ line at $71 \mu \mathrm{m})$ and also the SPIRE emission in the $5_{23}-5_{14}$ line at $212 \mu \mathrm{m}$ (although this line is only marginally detected at the $3 \sigma$ level). However, the core component predicts negligible emission in most SPIRE lines (and even absorption in the $2_{20}-2_{11}$ line), which indicates the presence of a more extended component $(R \sim 280 \mathrm{pc})$, moderately warm $\left(T_{\text {dust }} \sim 55 \mathrm{~K}\right)$, and with lower column $\left(\tau_{100 \mu \mathrm{m}} \sim 0.5\right)$. This extended component, mostly responsible for the $\mathrm{H}_{2} \mathrm{O}$ emission observed with SPIRE, is naturally identified with the inner disc traced by the $J=3-2$ lines of $\mathrm{HCO}^{+}$and $\mathrm{HCN}(\sim 300 \mathrm{pc})$.

The two water components together provide a good fit to the FIR emission, though the combined model underestimates, to some extent, the flux densities between 100 and $200 \mu \mathrm{m}$. This probably indicates a range in $T_{\text {dust }}$ for the disc component, rather than a single value. It is also worth noting that the source luminosity is dominated by the disc (i.e. the starburst), with the core component accounting for $L_{\mathrm{IR}} \sim 4 \times 10^{11} L_{\odot}$. The latter corresponds to $\sim 30 \%$ of the total IR luminosity, which is close to the estimated AGN contribution based on the 15-30 $\mu \mathrm{m}$ diagnostic (e.g. Veilleux et al. 2009). The blueshift of the absorption lines, which are dominated by the core component, is similar to that seen in the excited $\mathrm{OH}$ lines (González-Alfonso et al. 2017), suggesting that the core is expanding (more details in Sect. 4.3). Our model for the core also predicts significant emission at $265 \mathrm{GHz}, \sim 10 \mathrm{mJy}$, though this value is relatively uncertain because of its dependence on the actual continuum optical depth and the mass absorption coefficient of dust $\left(\kappa_{\lambda}\right)$ at millimetre wavelengths ${ }^{5}$.

\section{Discusion}

\subsection{A rotating disc with continuum absorption}

The integrated intensities, velocity fields, and $p-v$ diagrams of $\mathrm{HCN}$ and $\mathrm{HCO}^{+}$show the typical pattern of a rotating disc (Figs. 6, 8 and 9). Such a rotating body should in principle be reflected in the spectra as double-peaked lines. However, due to our relatively large beam size used to observe the $(1-0)$ lines $\left(4^{\prime \prime} .9 \times 44^{\prime \prime} 5\right)$, most of the gas is concentrated in the central pixel and the velocity gradients within the beam are not well reproduced. Therefore, our $\mathrm{HCN}$ and $\mathrm{HCO}^{+}(1-0)$ lines show a single Gaussian-like profile. On the other hand, the $(3-2)$ transitions were observed with a resolution significantly higher $\left(0^{\prime \prime} .61 \times 00^{\prime \prime} .55\right)$, so the velocity gradients are well traced and the spectral lines reveal the expected pattern of the inner rotating disc (see Sect. 3.3.1).

In addition, continuum absorption is also apparently contributing to the shape of the $\mathrm{HCN}$ and $\mathrm{HCO}^{+}(3-2)$ profiles. Firstly, we note that the $\sim 20-25$ mJy drop flux of the lines is very similar to the continuum flux density at these frequencies, of $29 \mathrm{mJy}$. Secondly, the velocity of the minimum flux, around $-50 \mathrm{~km} \mathrm{~s}^{-1}$, roughly coincides with the peak absorption velocities of the $\mathrm{H}_{2} \mathrm{O}$ lines observed with PACS, and of the $\mathrm{OH} 84 \mu \mathrm{m}$ and $\mathrm{OH} 65 \mu \mathrm{m}$ lines presented in González-Alfonso et al. (2017). The foreground warm gas traced by $\mathrm{H}_{2} \mathrm{O}$ and $\mathrm{OH}$ is absorbing the continuum (e.g. González-Alfonso et al. 2017), and the coincidence in velocities and continuum values of the dip in $\mathrm{HCN}$ and $\mathrm{HCO}^{+}$indicates that the dense gas might also be absorbing the background dust emission.

In this context, the two peaks of the $\mathrm{HCN}$ and $\mathrm{HCO}^{+}(3-2)$ lines are probing the edges of the inner rotating disc, while the absorbed flux indicates the positions of the maximum column densities of the gas. The channel maps shown in Figs. 13

\footnotetext{
5 For the core component, we modified the $k_{\lambda}$ curve in Fig. 2 of González-Alfonso et al. (2014) in such a way that $\kappa_{1.3 \mathrm{~mm}}=0.9 \mathrm{~cm}^{2} \mathrm{~g}^{-1}$ of dust, more similar to the value used by Downes \& Solomon (1998).
} 

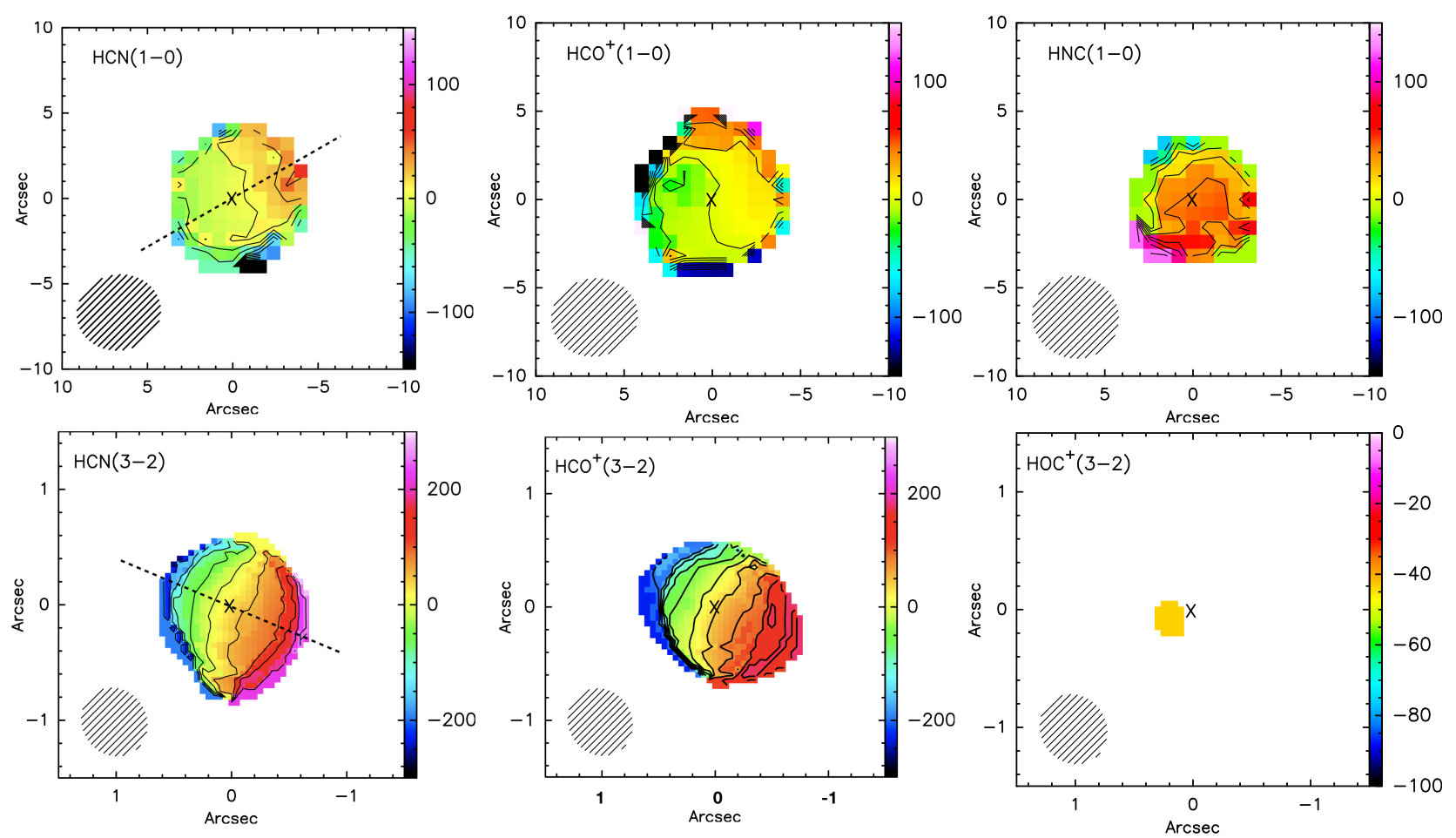

Fig. 8. Velocity fields (moment 1 maps). The coloured velocity scale (right axis) is in $\mathrm{km} \mathrm{s}^{-1}$. The step in contours is $20 \mathrm{kms}^{-1}$ for all lines. We note the blue-shifted velocities of $\operatorname{HOC}^{+}(3-2)$. The crosses in the centre of each panel indicate the position of the nuclear source. The beam is shown in the lower left corner of each panel. North is up, and east is to the left. The dashed lines in the HCN plots indicate the cut for the $p-v$ diagrams shown in Fig. 9.

and 14 also show a minimum emission around $-50 \mathrm{~km} \mathrm{~s}^{-1}$ and $-100 \mathrm{~km} \mathrm{~s}^{-1}$ (better seen in the $\mathrm{HCO}^{+}(3-2)$ map), velocities at which there is a maximum absorption of the continuum.

Self-absorption might also be an extra factor affecting the line shapes if there is cooler foreground gas with high-enough column densities. This, however, is difficult to disentangle from the continuum absorption in our data. In addition, it is possible that the opacity of the gas in the centre of Mrk 273 is high enough to result in flat-topped profiles such as some of those observed in the $\mathrm{HCN}$ and $\mathrm{HCO}^{+}(1-0)$ transitions, even though these species are less abundant than $\mathrm{CO}$. We note that any kind of absorption implies that our estimations of line fluxes, luminosities, and molecular gas mass are lower limits to the actual values.

\subsection{The Mrk273 molecular outflow}

As commented in Sect.3.2.1, all the water lines observed with Herschel/PACS are consistently blue-shifted with respect to the systemic velocity of Mrk 273. The absorption in the various lines peaks in the range $[-20,-140] \mathrm{km} \mathrm{s}^{-1}$ and extends as far as $-600 \mathrm{~km} \mathrm{~s}^{-1}$. Velocity shifts are also observed in $\mathrm{OH} 65 \mu \mathrm{m}$ and $\mathrm{OH} 84 \mu \mathrm{m}$ (González-Alfonso et al. 2017), and trace the low-velocity gas of the approaching component of the outflow (González-Alfonso et al. 2017).

The channel maps of $\mathrm{HCN}$ and $\mathrm{HCO}^{+}$(Figs. 11-14) show clear emission elongated to the north and south. This seems to correspond to the red-shifted low-velocity component $\left(\left|v-v_{\text {sys }}\right|<400 \mathrm{~km} \mathrm{~s}^{-1}\right)$ of the wind heading to the north observed with CO (Cicone et al. 2014). In addition, we also see emission in the north-south direction at higher velocities (up to $\sim 400 \mathrm{~km} \mathrm{~s}^{-1}$ ). In our data, we distinguish two velocity compo- nents of the outflow: one with relatively low velocities $(\mid v-$ $v_{\text {sys }} \mid<400 \mathrm{~km} \mathrm{~s}^{-1}$ ) that is seen only in the channel maps (because its emission is blended with that of the disc in the spectra); and a high-velocity component $\left(\left|v-v_{\text {sys }}\right|>400 \mathrm{~km} \mathrm{~s}^{-1}\right)$ that is seen in the spectrum of the central channel as a blue-shifted shoulder of the $\operatorname{HCN}(3-2)$ line (Fig. 5). The HCN $(3-2)$ spectral bump spans approximately from $-400 \mathrm{~km} \mathrm{~s}^{-1}$ to $-1000 \mathrm{~km} \mathrm{~s}^{-1}$ (when centring the line at the rest frequency of $\operatorname{HCN}(3-2)$ ), which is consistent with the outflow velocities measured with CO (Cicone et al. 2014).

As explained in Sect. 3.3.2, the size of the fast wind traced by $\operatorname{HCN}(3-2)$ is $0 .{ }^{\prime \prime} 61 \pm 0 .{ }^{\prime \prime} 05(\sim 460 \mathrm{pc})$ and the elongated shape towards the north can be seen clearly (Fig. 7). The $\mathrm{CO}(1-0)$ outflowing gas extends up to $550-600 \mathrm{pc}$ in Mrk 273 (Cicone et al. 2014). This difference suggests that the expelled moderate-density gas $\left(n_{\mathrm{H}_{2}} \leq 10^{3} \mathrm{~cm}^{-3}\right)$ travels further than the dense gas $\left(n_{\mathrm{H}_{2}} \geq 10^{4} \mathrm{~cm}^{-3}\right)$. In the right panel of Fig. 16, we show a sketch of the dense and warm outflow properties derived from our observations of Mrk 273, as well as the comparison with the diffuse phase of the outflow detected in $\mathrm{CO}$ by Cicone et al. (2014).

The $\mathrm{HCN}$ fast outflow luminosity is $L_{\mathrm{HCN}(3-2)}^{\text {outflow }}=\pi R^{2} I=$ $8 \times 10^{7} \mathrm{~K} \mathrm{~km} \mathrm{~s}^{-1} \mathrm{pc}^{2}$. To estimate the dense gas mass contained in the outflow, we used the relation $M_{\text {dense }}=10 \times L^{\prime}[\mathrm{HCN}(3-2)]$ (Gao \& Solomon 2004). We note that this formula might overestimate the actual value, because it assumes that all gas is virialised, which might not be the case in the outflow. Additionally, the $\mathrm{HCN}-$ to- $\mathrm{H}_{2}$ conversion factor, which is not well constrained in ULIRGs, could also lead to an overestimation of the gas mass. A detailed analysis of the factors affecting the gas mass estimation can be found in Gao \& Solomon (2004). We obtain $M_{\text {dense }}^{\text {outllow }} \leq 8 \times 10^{8} M_{\odot}$, which is consistent with the value obtained 

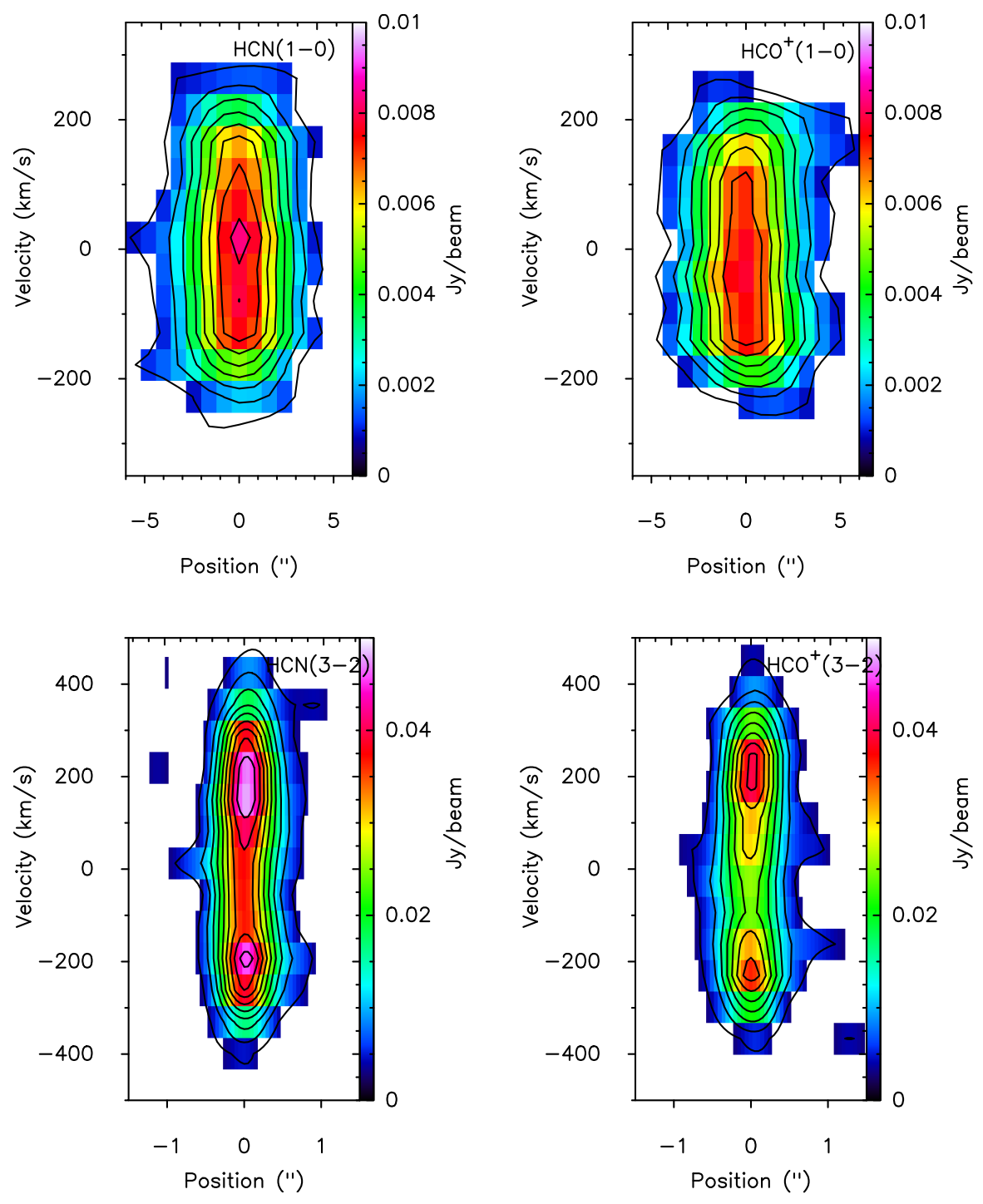

Fig. 9. Position-velocity (p-v) maps of $\mathrm{HCN}$ and $\mathrm{HCO}^{+}$. The cuts along the axes of rotation are shown by the dashed lines in Fig. 8. Contour steps of the $(1-0)$ maps go from

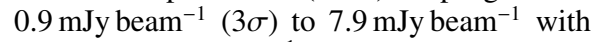
steps of $1 \mathrm{mJy}^{-1}$ beam $^{-1}$. For the $(3-2)$ maps, contours go from $3.9 \mathrm{mJybeam}^{-1}(3 \sigma)$ to $59 \mathrm{mJy}$ beam ${ }^{-1}$ with steps of $5 \mathrm{mJy}$ beam $^{-1}$.

from $\mathrm{OH}$ observations $\left(\sim 1.6 \times 10^{8} M_{\odot}\right.$, González-Alfonso et al. 2017), and is also similar to the dense gas mass in the outflow of Mrk $231\left(\sim 4 \times 10^{8} M_{\odot}\right.$, Aalto et al. 2015a). We note that our result refers only to the high-velocity gas $\left(\left|v-v_{\text {sys }}\right|>400 \mathrm{~km} \mathrm{~s}^{-1}\right)$, as it is not possible to separate the slow component of the outflow from the disc emission in the HCN data.

In cases where the outflow and the disc kinematics cannot be distinguished well in the velocity maps (as in our Fig. 8), a difference in their position angles can help to probe the kinematic decoupling between the two, and can also yield information about the nuclear powering source. Gas in starburst-powered outflows is always expelled perpendicular to the plane of the galaxy (thus showing a change of $90^{\circ}$ with respect to the PA of the starbursting disc), while AGN-powered outflows might have virtually any position angle, because the dusty torus and the accretion disc can be tilted with respect to the disc (García-Burillo et al. 2015). In Mrk 273, we measure a difference between the PA of the inner disc (of $71^{\circ} \pm 5^{\circ}$, from $\mathrm{HCO}^{+}(3-2)$, Table 3 ) and the PA of the outflow $\left(10^{\circ} \pm 3^{\circ}\right)$, of $60^{\circ} \pm 8^{\circ}$. This seems to indicate that the outflow is powered by the central AGN. Indeed, Cicone et al. (2014) found that the high outflow mass-loss rate of Mrk 273 is consistent with the linear correlation they observed between the bolometric AGN luminosity and the mass outflow rate in local AGN-host galaxies.
A couple of interesting issues arising from our data are 1) the red-shifted component of the outflow traced by $\mathrm{HCN}(3-2)$ is seen only at low velocities in the channel maps $\left(\left|v-v_{\text {sys }}\right|<400 \mathrm{~km} \mathrm{~s}^{-1}\right)$. Indeed, a fast red-shifted wind, which should be visible in the spectra (with a similar intensity as the blue-shifted one), does not appear; and 2) the apparent absence of high-velocity winds in $\mathrm{HCO}^{+}$(i.e. $\mathrm{HCO}^{+}$line wings at $\left|v-v_{\text {sys }}\right|>400 \mathrm{~km} \mathrm{~s}^{-1}$ ). Regarding the first issue, deeper observations are required to improve the $\mathrm{S} / \mathrm{N}$ of the outflow, to make sure that the red-shifted component is not present. If that were the case, then it would imply that either there is a density or temperature gradient between the receding and the approaching gas (with the red-shifted gas being less dense and/or warm in general), or there is a chemical differentiation between the two. These are not uncommon characteristics in galactic winds, such as in Mrk 231 (Aalto et al. 2012, 2015a). The chemical differentiation could also explain why there is not enough high-velocity gas traced by $\mathrm{HCO}^{+}$in the outflow (see Lindberg et al. 2016 for details about this phenomena in the wind of Mrk 231).

\subsection{Expansion of the core}

In Mrk 273, the velocity peaks of the $\mathrm{OH} 65 \mu \mathrm{m}$ and OI $63 \mu \mathrm{m}$ lines are shifted in comparison to the emission peak of [CII] 

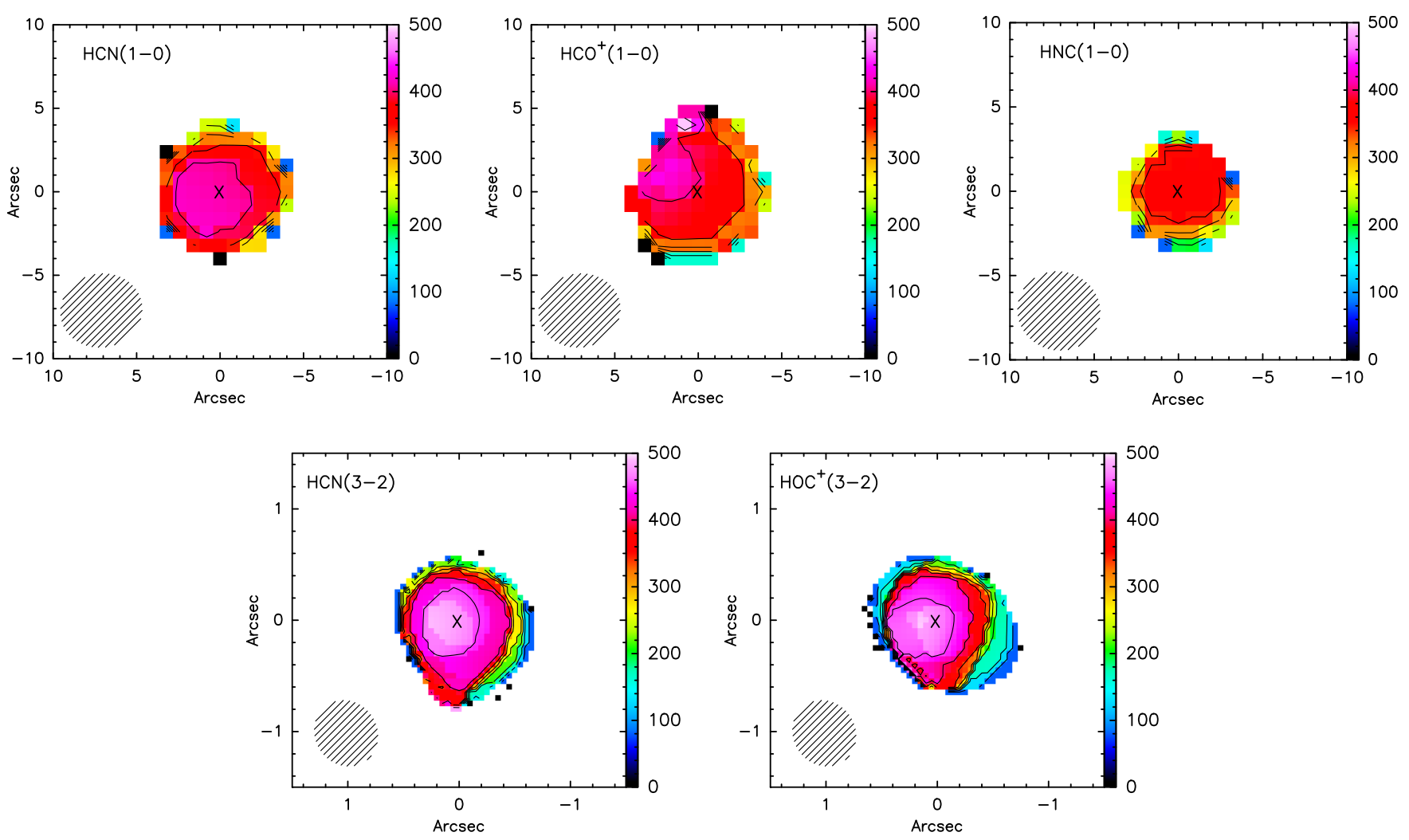

Fig. 10. Velocity dispersions. Contours go from 0 to $500 \mathrm{kms}^{-1}$ with steps of $50 \mathrm{kms}^{-1}$ for all lines. The crosses in the centre of each panel indicate the position of the nuclear source. The synthesised beam is shown in the bottom-left corner of each panel. North is up and East is to the left.

at $158 \mu \mathrm{m}$ (González-Alfonso et al. 2017). The [CII] maximum is found at zero velocities (with respect to the systemic velocity of the galaxy), and arises from the bulk of the warm gas in the nucleus. The $\mathrm{OH} 65 \mu \mathrm{m}$ and $\mathrm{OI} 63 \mu \mathrm{m}$ lines, however, have their maximum (absorption/emission) peaks shifted by $\pm 50 \mathrm{~km} \mathrm{~s}^{-1}$, and trace the blue component of the outflowing gas moving at low velocities. Apart from being a sign of a superwind, González-Alfonso et al. (2017) argue that this change in the redshift also indicates that the large columns of gas close to the central engine are expanding at low velocities. This effect has been found in a number of other ULIRGs where low-velocity outflows are also present (González-Alfonso et al. 2017).

As discussed in Sect.4.1, the shift of approximately $50 \mathrm{~km} \mathrm{~s}^{-1}$ seen in $\mathrm{OH} 65 \mu \mathrm{m}$ and OI $63 \mu \mathrm{m}$ also appears in our $\mathrm{H}_{2} \mathrm{O}$ data. In particular, the $\mathrm{H}_{2} \mathrm{O}$ lines observed with PACS, which according to our models trace the compact core with a radius $\sim 50 \mathrm{pc}$, are also blue-shifted with respect to [CII] by $[-20,-140] \mathrm{km} \mathrm{s}^{-1}$ (Table 2). This indicates that, apart from rotating, the gas in the core is expanding at low velocities, pushing outwards the boundaries with the inner disc traced by the HCN and $\mathrm{HCO}^{+}(3-2)$ lines. Indeed, the expansion is also reflected in our $\mathrm{HCO}^{+}$and $\mathrm{HCN}(3-2)$ data. While $\mathrm{HCO}^{+}$and $\mathrm{HCN}(3-2)$ double-peaks probe the outer edges of the inner disc (at a radius of $\sim 300 \mathrm{pc}$ ), their peaks of absorption probe the kinematics of the bulk of the disc, which is also shifted by $50-100 \mathrm{~km} \mathrm{~s}^{-1}$. We interpret this as the gas in the inner disc being pushed by the expanding gas of the core. We illustrate this in the sketch shown in the right panel of Fig. 16.

\subsection{Non-detection of vibrational emission}

Rotational transitions within vibrationally excited levels of $\mathrm{HCN}$ and $\mathrm{HC}_{3} \mathrm{~N}$ have been observed in the central $<100 \mathrm{pc}$ of several (U)LIRGs, probing regions of high temperatures of $\geq 100 \mathrm{~K}$ (Sakamoto et al. 2010; Costagliola et al. 2015; Aalto et al. 2015b; Martín et al. 2011, 2016), and high column densities. Their excitation cannot be explained by collisional effects alone, and mid-IR pumping is necessary in order to populate the upper energy levels and fit their observed luminosities (Aalto et al. 2015b). In that case, the vibrational lines may be more suitable to study the optically thick dust cores of galaxies than the rotational transitions (see Aalto et al. 2015b for a detailed discussion).

In our NOEMA observations of Mrk 273, we do not detect the vibrationally excited line $\operatorname{HCN}(3-2) v_{2}=1$. The line is split into two components with energy levels of similar intensity, v2 $=1$ e and v2 = 1f, at frequencies 265.8 and $267.2 \mathrm{GHz}$. The first one is completely blended with the $\operatorname{HCN}(3-2)$ rotational line due to the galactic broad line widths, and it is not possible to estimate its peak temperature. However, the $\mathrm{v}_{2}=1 \mathrm{f}$ transition would appear as a bump only partially blended with the red side of the $\mathrm{HCO}^{+}(3-2)$ transition. We calculate a limit to its flux density in the central pixel of $1.1 \mathrm{mJy}$, which is slightly below the $1 \sigma$ rms level (of $1.2 \mathrm{mJy}$, calculated at the final velocity resolution). Assuming that the vibrational line has the same FWHM as the HCN rotational transition $\left(\sim 600 \mathrm{~km} \mathrm{~s}^{-1}\right)$, the limit to the integrated intensity was estimated as $I \leq 3 \times r m s \times \sqrt{F W H M \times D v}=4 \mathrm{~K} \mathrm{~km} \mathrm{~s}^{-1}(D v$, the final spectral resolution, is $68 \mathrm{~km} \mathrm{~s}^{-1}$ ). Under the conservative assumption that both rotational and vibrational lines have the same source size, the upper limit to the HCN-vib luminosity is $L^{\prime}=\pi R^{2} I \leq 7.3 \times 10^{5} \mathrm{~K} \mathrm{~km} \mathrm{~s}^{-1} \mathrm{pc}^{2}$ ( $R$ is the source size of the $\operatorname{HCN}(3-2)$ emission specified in Table 3$)$. This is at least a factor of two fainter than the $L_{\mathrm{HCN}_{\mathrm{vib}}(3-2)}^{\prime}$ in Mrk $231\left(1.7 \times 10^{7} \mathrm{~K} \mathrm{~km} \mathrm{~s}^{-1} \mathrm{pc}^{2}\right.$, Aalto et al. 2015a). This is 


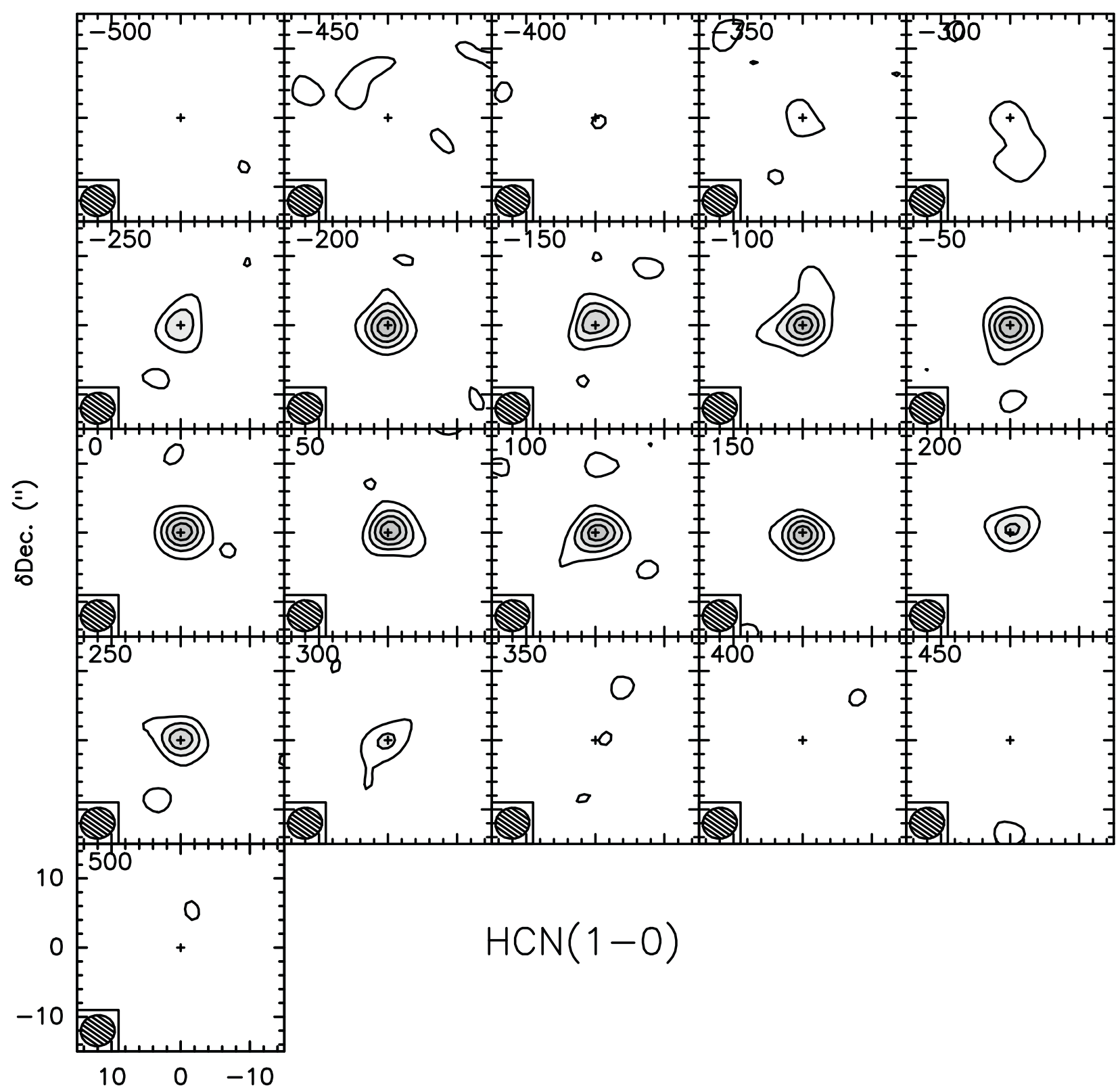

SR.A.(")

Fig. 11. Channel-velocity maps of $\mathrm{HCN}(1-0)$ in the velocity range $[-500,500] \mathrm{km} \mathrm{s}^{-1}$ with steps of $50 \mathrm{~km} \mathrm{~s}^{-1}$. Contours go from $1.5 \mathrm{mJy}$ beam ${ }^{-1}$ $(5 \sigma)$ to $9.5 \mathrm{mJy}_{\text {beam }}^{-1}$ with a spacing of $2 \mathrm{mJy}_{\text {beam }}^{-1}$. The synthesised beam is plotted in the bottom-left corner. North is up and east is to the left.

interesting, because Lahuis et al. (2007) detect the $14 \mu \mathrm{m} \mathrm{HCN}$ vib lines in absorption at the same level in both Mrk 273 and Mrk 231

Aalto et al. (2015b) found a tentative correlation between the outflow velocity and the intensity of HCN vibrationally excited lines in a moderate sample of ten (U)LIRGs. Galaxies with fast outflows (i.e. when the outflow velocity exceeds the escape velocity of the nuclear region) tend to have fainter vibrational lines. With its non detection of $\operatorname{HCN}(3-2) v_{2}=1$, and its fast molecular outflow, Mrk 273 also follows this relationship. The reason for this correlation, if true, requires further investigation.

Similarly, we do not detect the vibrationally excited line $\mathrm{HC}_{3} \mathrm{~N}(10-9) v_{6}=1$, even though the energy of its lower level is below that of the HCN-vib lines (Table 7). We obtain an upper limit to the peak flux density of $0.3 \mathrm{mJy}$. This line was first tentatively detected in the LIRG NGC 4418 with the IRAM 30 m telescope (Aalto et al. 2007; Costagliola \& Aalto 2010) and later confirmed by ALMA observations (Costagliola et al. 2015) with a rotational-to-vibrational intensity ratio of $\sim 11$. If we extrapolate that ratio to Mrk 273, then, based on our results, one would expect a flux density of $\sim 0.2 \mathrm{mJy}$. Under the assumption that, in Mrk 273, $\mathrm{HC}_{3} \mathrm{~N}(10-9) v_{6}=1$ likely arises from a region smaller than $\mathrm{HCN}(1-0)\left(\right.$ i.e. $\left.\theta<2 .^{\prime \prime} 0 \times 1 .^{\prime \prime} 6\right)$, the limit to its luminosity is $6 \times 10^{6} \mathrm{~K} \mathrm{~km} \mathrm{~s}^{-1} \mathrm{pc}^{2}$.

Apart from sensitivity issues, two possibilities can potentially explain why we do not detect vibrationally excited emission of $\mathrm{HCN}$ and $\mathrm{HC}_{3} \mathrm{~N}$ in Mrk 273. One is that the mid-IR radiation density in the core of Mrk 273 is not as strong as in other galaxies hosting compact obscured nuclei, such as Mrk 231, Arp 220 or NGC 4418. The other is that, although the mid-IR radiation is 


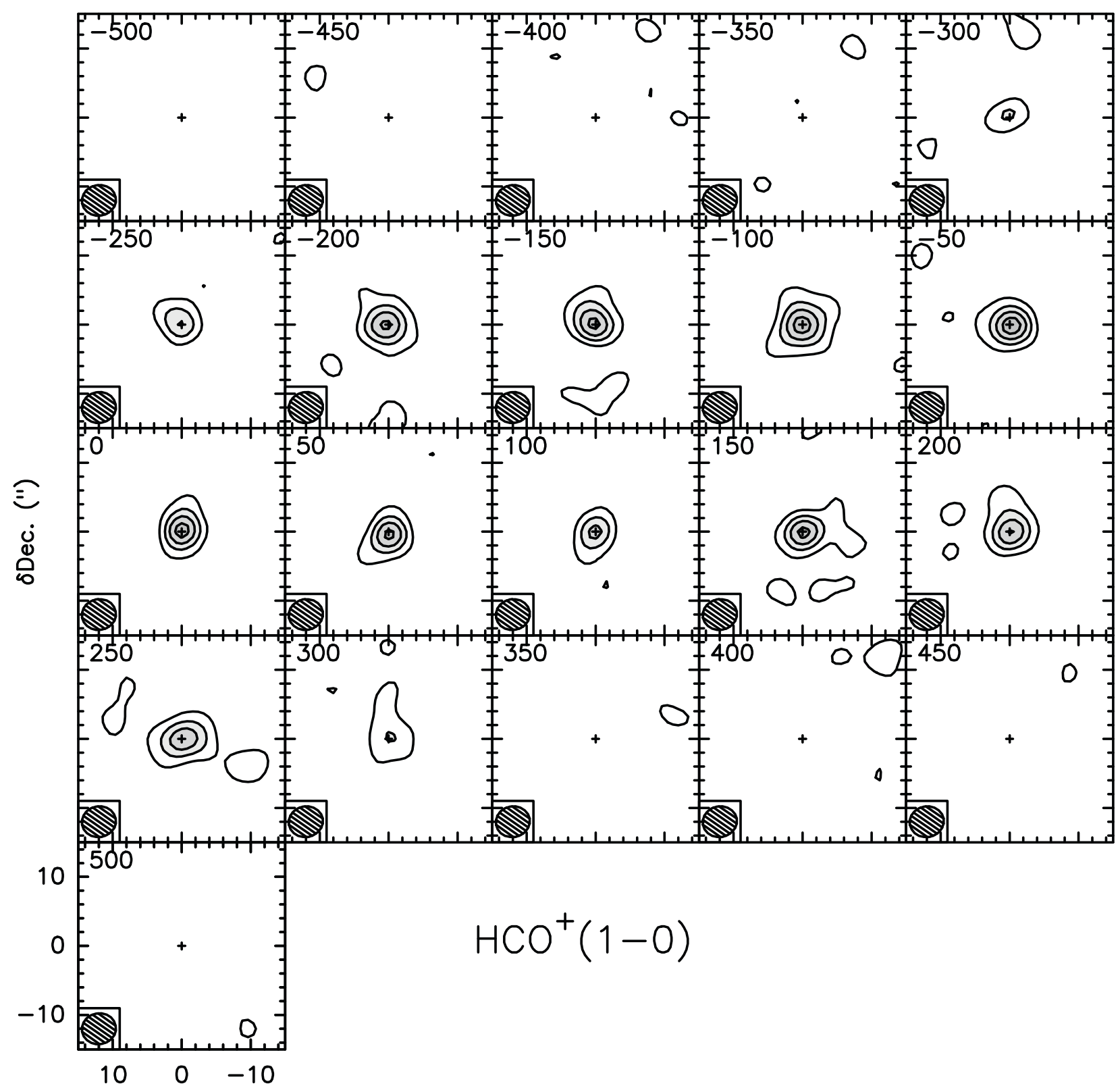

SR.A.(")

Fig. 12. Channel-velocity maps of $\mathrm{HCO}^{+}(1-0)$ in the velocity range $[-500,500] \mathrm{km} \mathrm{s}^{-1}$ with steps of $50 \mathrm{~km} \mathrm{~s}^{-1}$. Contours go from $1.5 \mathrm{mJy}$ beam ${ }^{-1}$ $(5 \sigma)$ to $9.5 \mathrm{mJy}_{\text {beam }}^{-1}$ with a spacing of $2 \mathrm{mJy}_{\text {beam }}^{-1}$. The synthesised beam is plotted in the bottom-left corner. North is up and east is to the left.

strong, the obscuration in the centre of Mrk 273 is so high that even the emission of vibrationally excited lines is extinguished.

\subsection{Molecular line ratios}

Tables 5 and 6 list the brightness temperature ratios of the species detected with NOEMA. The ratios were obtained by comparing the temperatures of the lines calculated over the entire emission (Table 5) and in the central pixel (Table 6). We note that the $3 \mathrm{~mm}$ lines $\left(\mathrm{HCN}, \mathrm{HCO}^{+}, \mathrm{HNC}(1-0)\right.$ and $\left.\mathrm{HC}_{3} \mathrm{~N}(10-9)\right)$ and the $1 \mathrm{~mm}$ lines $\left(\mathrm{HCN}, \mathrm{HCO}^{+}, \mathrm{HOC}+(3-2)\right)$ were observed with angular resolutions that differ in the covering area by a factor $>60$. In order to avoid confusion and unphysical results, we only discuss ratios in which both lines were observed with the same resolution. In the following we discuss the most relevant ratios.

\subsection{1. $\mathrm{HCN} / \mathrm{HCO}^{+}$}

The integrated intensity ratio between $\mathrm{HCN}$ and $\mathrm{HCO}^{+}$has been widely used to discriminate between starburst-powered galaxies and AGNs (Kohno et al. 2001; Krips et al. 2008; Imanishi et al. 2009; Izumi et al. 2013, 2016; Martín et al. 2015). An enhancement of the $\mathrm{HCN}$ abundance with respect to $\mathrm{HCO}^{+}$is observed in a fairly large sample of Seyferts, which show a $\mathrm{HCN} / \mathrm{HCO}^{+}$ ratio $\geq 1$. Starbursts, on the other hand, are found to have values $\leq 1$. The coexistence of both phenomena in the central few hundred parsecs can however lead to outlier values of the ratio if they are not resolved (Aladro et al. 2015; Privon et al. 2015).

The reason why the $\mathrm{HCN} / \mathrm{HCO}^{+}$ratio is a diagnostic of the galactic central activity is still debated. For more than a decade, the HCN enhancement was claimed to be due to X-ray irradiation in the vicinity of the SMBHs (e.g. Kohno et al. 2001). 


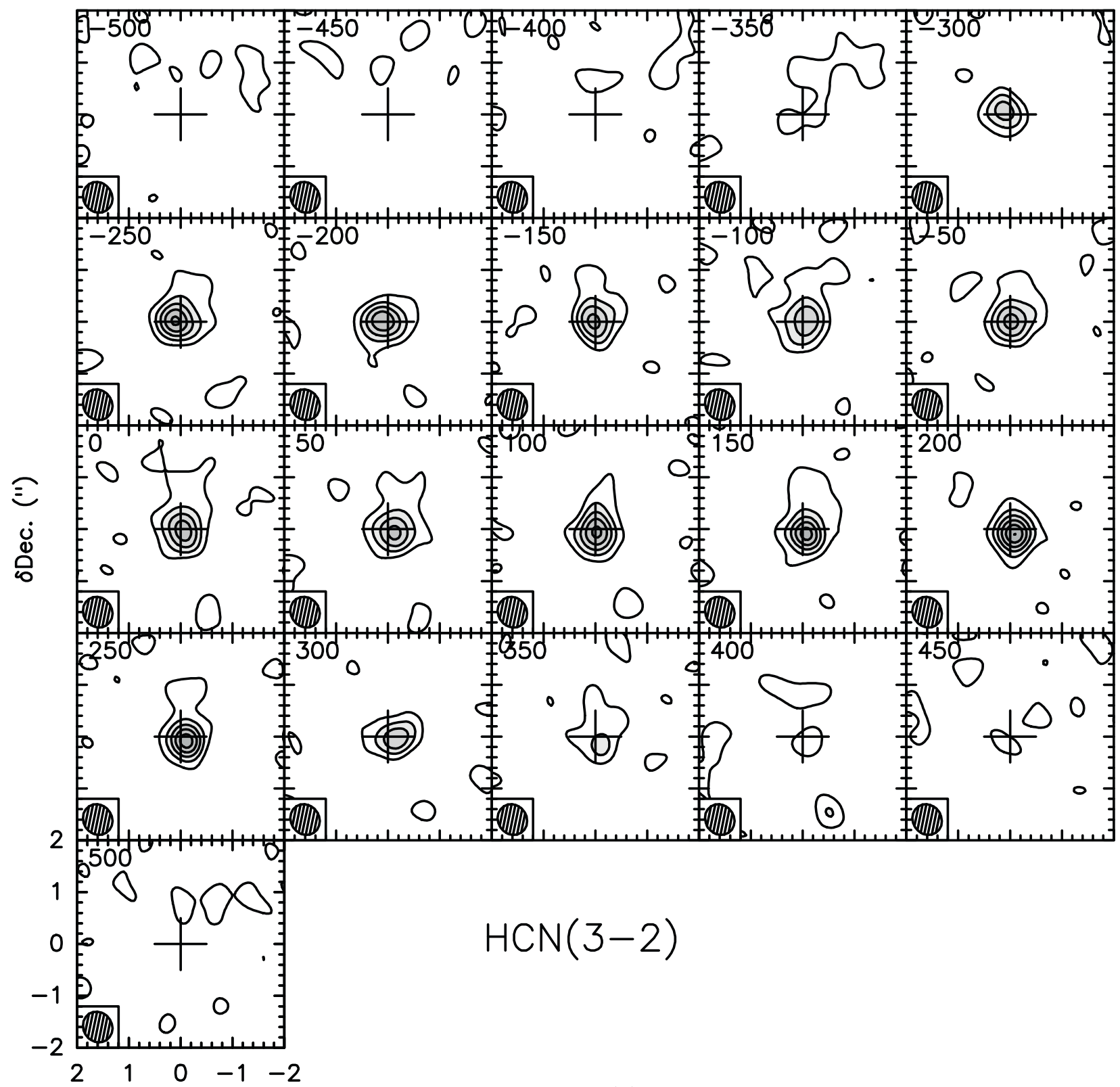

SR.A.(")

Fig. 13. Channel-velocity maps of $\mathrm{HCN}(3-2)$ in the velocity range $[-500,500] \mathrm{km} \mathrm{s}^{-1}$ with steps of $50 \mathrm{~km} \mathrm{~s}^{-1}$. Contours go from $6.5 \mathrm{mJy}$ beam ${ }^{-1}$ $(5 \sigma)$ to $56 \mathrm{mJy}_{\text {beam }}{ }^{-1}$ with a spacing of $10 \mathrm{mJy}_{\text {beam }}{ }^{-1}$. We highlight the significantly smaller spatial scale relative to the channel map of the $(1-0)$ line shown in Fig. 11. The synthesised beam is plotted in the bottom-left corner. North is up and east is to the left.

However, recent studies of NGC 1097 point to high temperatures and/or mechanical heating as a more plausible explanation (Martín et al. 2015; Izumi et al. 2016). Systematic high-angularresolution observations of a larger sample of galaxies/AGNs is still needed to clarify this issue.

In the case of $\mathrm{HCO}^{+}$, both observations and models point to an enhancement in starburst galaxies, and in particular in photondominated regions (PDRs), as a result of strong UV irradiation. Unfortunately, the scenario could be more complex, since chemical models suggest that $\mathrm{HCO}^{+}$can also be enhanced in regions heavily pervaded by cosmic ray/X-ray fields, such as in AGNs (Meijerink et al. 2006; Aladro et al. 2013). Yet this scenario has not been confirmed with observations, to our knowledge.

Bearing in mind that the reasons for the enhancements of $\mathrm{HCN}$ and $\mathrm{HCO}^{+}$are difficult to assess, the fact is that this ratio diagnoses the power sources of many well-known active galaxies. Nevertheless, there are some sources which do not seem to be consistent with this scenario. For example, some apparent starbursts have $\mathrm{HCN}$-to- $\mathrm{HCO}^{+}$ratios as high as AGNs, indicating that the HCN enhancement cannot be exclusively attributed to AGNs (Privon et al. 2015).

In Mrk 273, we measure the global brightness intensity ratios $\mathrm{HCN}(1-0) / \mathrm{HCO}^{+}(1-0)=1.0 \pm 0.2$ and $\mathrm{HCN}(3-2) / \mathrm{HCO}^{+}(3-2)=1.1 \pm 0.3 \quad$ (Table 5). Evaluating the nuclear ratios in the central pixel, gives very similar values of $1.0 \pm 0.2$ and $1.3 \pm 0.3$ for the $(1-0)$ and $(3-2)$ transitions respectively (Table 6). These ratios place the galaxy in an ambiguous region of the diagnostic diagram, where a starburst, an AGN, or a combination of the two are possible. Depending on the diagnostic used in the literature, either an AGN or a 


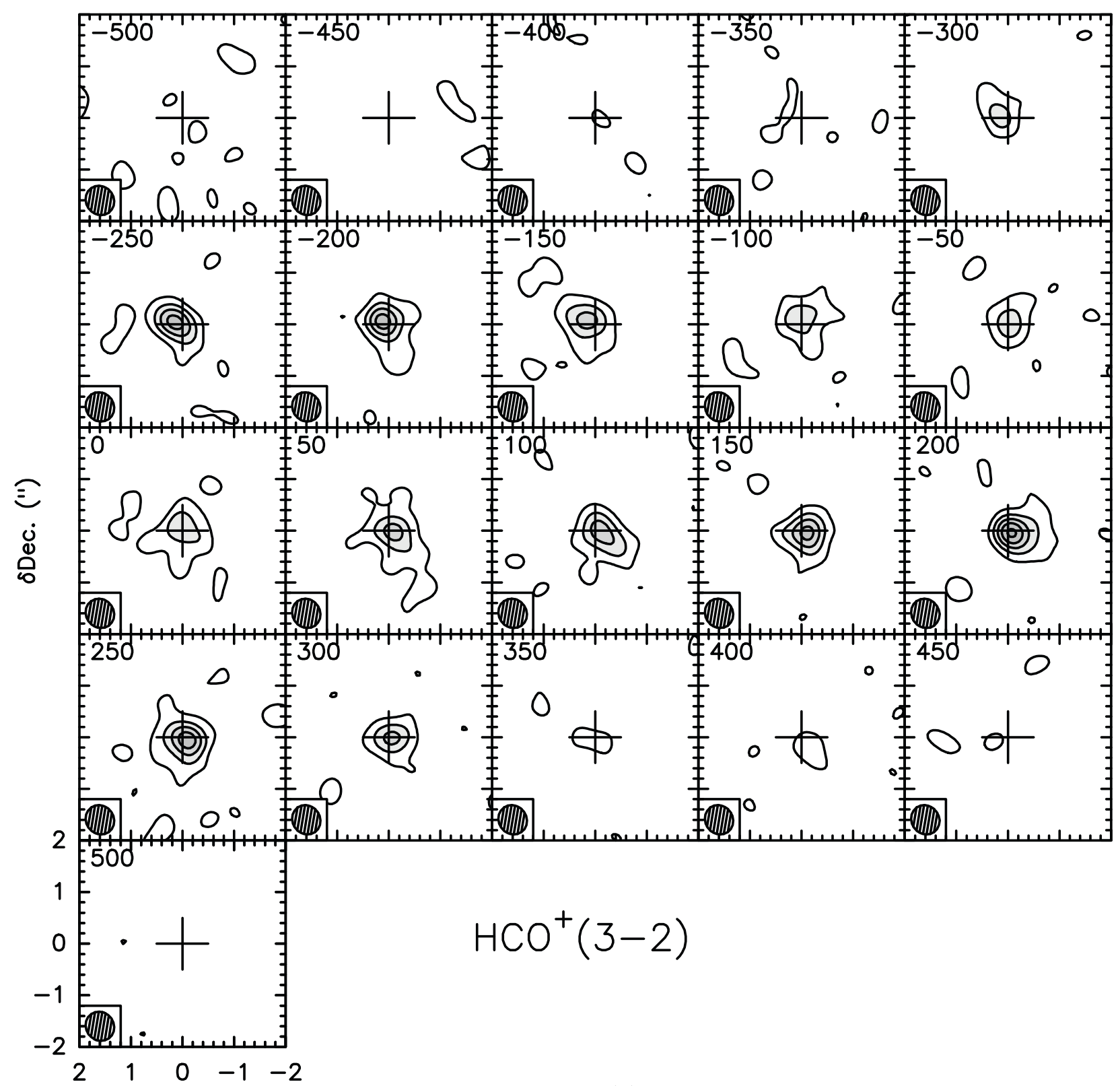

$\delta$ R.A.(")

Fig. 14. Channel-velocity maps of $\mathrm{HCO}^{+}(3-2)$ in the velocity range $[-500,500] \mathrm{km} \mathrm{s}^{-1}$ with steps of $50 \mathrm{~km} \mathrm{~s}^{-1}$. Contours go from $6.5 \mathrm{mJy}$ beam ${ }^{-1}$ $(5 \sigma)$ to $56 \mathrm{mJy}_{\text {beam }}{ }^{-1}$ with a spacing of $10 \mathrm{mJy}_{\text {beam }}^{-1}$. We highlight the significantly smaller spatial scale relative to the channel maps of the $(1-0)$ line shown in Fig. 12. The synthesised beam is plotted in the bottom-left corner. North is up and east is to the left.

starburst are claimed to power the northern nucleus of Mrk 273. Perhaps a mixture of both activities are present in the central hundred parsecs. Unfortunately, our $\mathrm{HCN} / \mathrm{HCO}^{+}$ratio does not help to elucidate the nature of the Mrk 273 nuclear source. Nevertheless, we note that both lines are significantly affected by opacity (Sect. 4.1), and that a more thorough analysis of this ratio including obscuration effects could further elucidate our results.

Using the Nobeyama Millimeter Array and RAINBOW interferometers at the Nobeyama Radio Observatory ${ }^{6}$, Imanishi et al. (2006) obtained a flux ratio $\mathrm{HCN}(1-0) / \mathrm{HCO}^{+}(1-0)>1.8$ in Mrk 273, within a beam of 1 '!9 91 '"5. This was interpreted as a signature of an AGN-dominated nucleus. This lower limit is much higher than our values, but we note that the latter authors did not

6 https://www.nro.nao.ac.jp/en/astronomer/ detect $\mathrm{HCO}^{+}(1-0)$, and that their $\mathrm{HCN}(1-0)$ and continuum fluxes are $70-80 \%$ lower than our values.

\subsection{2. $\mathrm{HCN} / \mathrm{HNC}$ and $\mathrm{HCO}^{+} / \mathrm{HNC}$}

Some (U)LIRGs, such as Arp 220 and Mrk 231, are extremely bright in $\mathrm{HNC}$, with line intensities almost equal to or even surpassing those of HCN (Huettemeister et al. 1995; Aalto et al. 2002; Aladro et al. 2015). Several scenarios can produce this unusually high line ratio, such as chemical reactions at moderate densities and temperatures $\left(n \sim 10^{4}-10^{5} \mathrm{~cm}^{-3}, T_{\text {kin }} \sim 50 \mathrm{~K}\right)$, high opacities of $\mathrm{HCN}$ with respect to $\mathrm{HNC}$ and, very likely, infrared pumping affecting HNC more than HCN (Aalto et al. 2002). The $\mathrm{HCN} / \mathrm{HNC}$ intensity ratio can reflect the evolutionary stage of starburst regions in galactic centres, with faint $\mathrm{HNC}$ emission 
R. Aladro et al.: Molecular gas in the northern nucleus of Mrk 273: Physical and chemical properties of the disc and its outflow
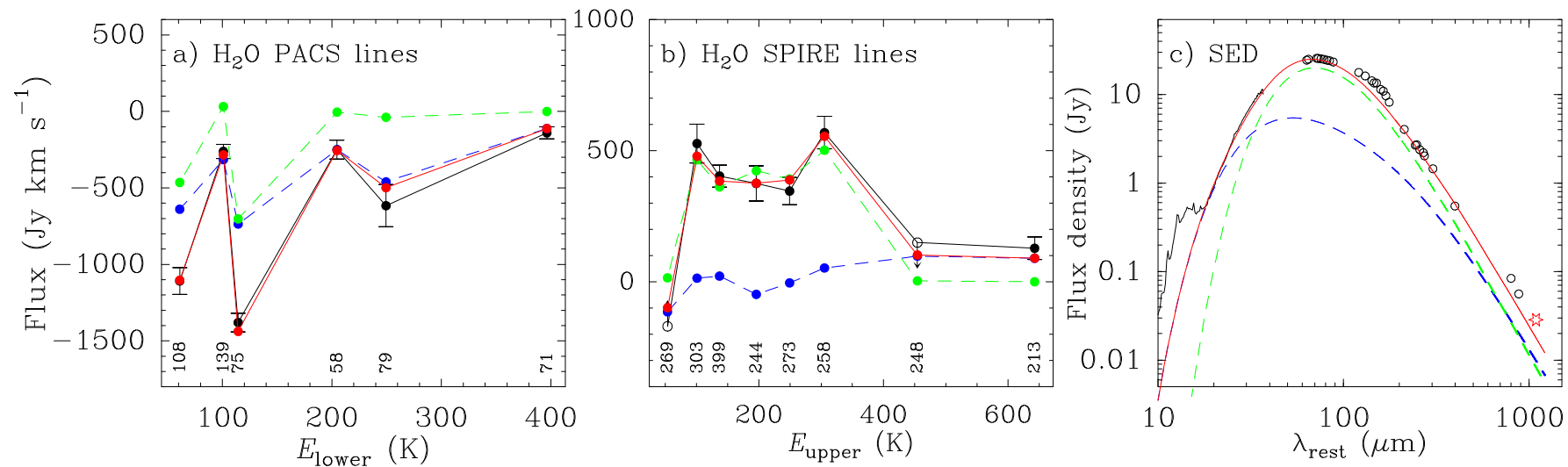

Fig. 15. Comparison between the observed (black symbols) and modelled (coloured symbols and lines) $\mathrm{H}_{2} \mathrm{O}$ fluxes in Mrk 273 within panel $a$ : PACS and panel $b$ : SPIRE. The model includes two components: the core (in blue), which accounts for most absorption lines observed within PACS, and the inner disc (in green), which dominates the emission of the sub-mm lines with $E_{\text {upper }}<400 \mathrm{~K}$ observed with SPIRE (see Table 4). Red colours and symbols indicate total modelled fluxes. The numbers at the bottom of panels a and $b$ indicate rounded-up transition wavelengths in $\mu \mathrm{m}$. Panel $c$ : the SED of Mrk 273, including the Spitzer/IRS spectrum, Herschel/PACS and SPIRE continuum data from observations of both $\mathrm{H}_{2} \mathrm{O}$ and $\mathrm{OH}$ lines, sub-mm data at 800 and $880 \mu \mathrm{m}$ (Rigopoulou et al. 1996; Wilson et al. 2008), and our measured flux density at $1 \mathrm{~mm}$ (starred-red symbol), is compared with the prediction of our composite model.

Table 4. Results for the two-component modelling of the $\mathrm{H}_{2} \mathrm{O}$ lines in Mrk 273.

\begin{tabular}{lcccccc}
\hline \hline Component & $\begin{array}{c}T_{\text {dust }} \\
(\mathrm{K})\end{array}$ & $\tau_{100 \mu \mathrm{m}}$ & $\begin{array}{c}R \\
(\mathrm{pc})\end{array}$ & $\begin{array}{c}L_{\mathrm{IR}} \\
\left(10^{11} L_{\odot}\right)\end{array}$ & $\begin{array}{c}N\left(\mathrm{H}_{2} \mathrm{O}\right) \\
\left(10^{16} \mathrm{~cm}^{-2}\right)\end{array}$ & $\begin{array}{c}X\left(\mathrm{H}_{2} \mathrm{O}\right) \\
\left(10^{-6}\right)\end{array}$ \\
\hline Core & $95(\gtrsim 80)$ & $5.0(\gtrsim 4)$ & $51(40-60)$ & $3.8(2.3-5.0)$ & $800(\gtrsim 400)$ & $1.2(0.6-3)$ \\
Inner disc & $55(45-65)$ & $0.5(0.2-1)$ & $284(180-360)$ & $8.0(6-12)$ & $7.8(5-20)$ & $0.12(0.08-0.5)$ \\
\hline
\end{tabular}

Notes. Numbers in parenthesis indicate the most plausible ranges, as inferred from all combinations with $\chi^{2}$ not exceeding $1.7 \times$ the minimum value.
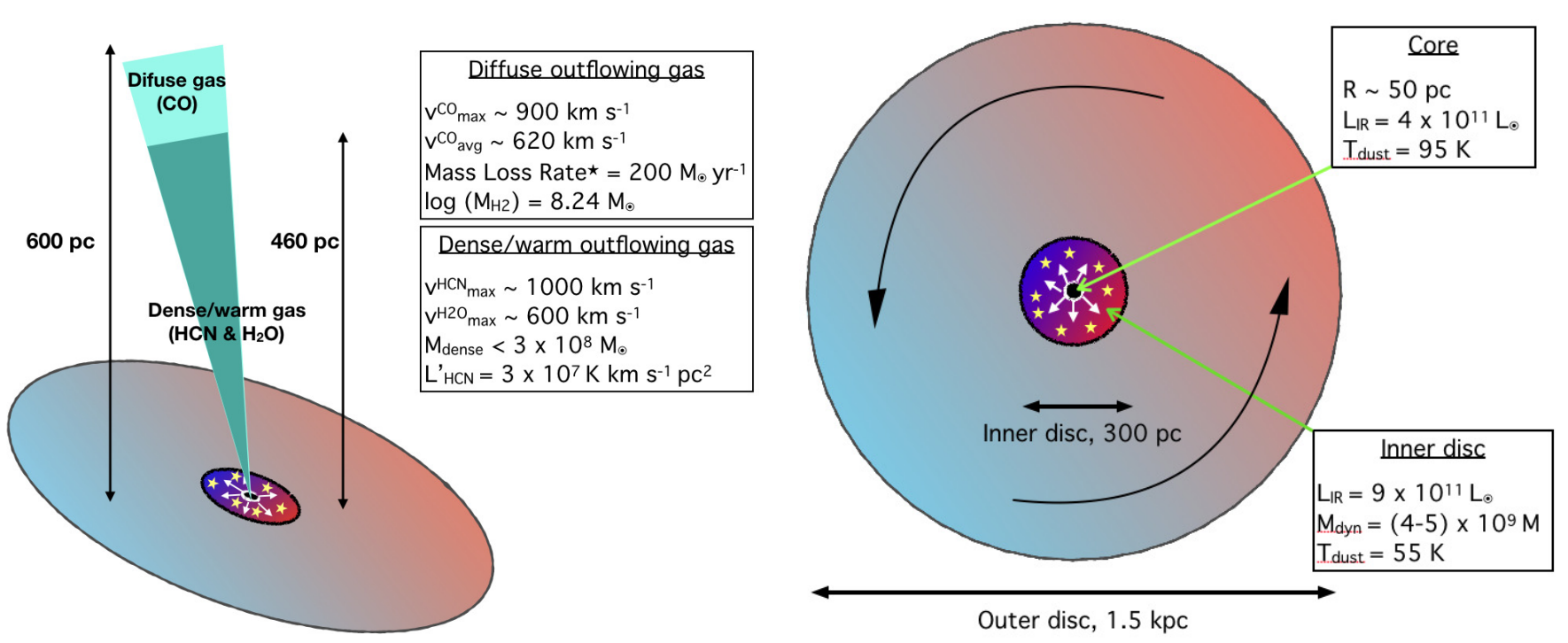

Fig. 16. Left: sketch of the blue-shifted molecular outflow in the northern nucleus of Mrk 273. The dense and warm outflowing gas found in our $\mathrm{HCN}$ and $\mathrm{H}_{2} \mathrm{O}$ data, and its properties, is compared to the diffuse outflow found by Cicone et al. (2014) by observing CO $(1-0)$. ${ }^{\star}$ We note that the mass loss rate is calculated as $\dot{\mathrm{M}}=v\left(M_{\mathrm{OF}} / R_{\mathrm{OF}}\right)$ (see Sect. 5.5 in González-Alfonso et al. 2017), which is a factor of three lower than the value given by Cicone et al. (2014). The position angles of the outflow $\left(10^{\circ}\right)$ and the inner disc $\left(70^{\circ}\right)$ are represented. Right: a face-on sketch of the three disc components identified in our data (outer disc, inner disc, and core), which are plotted in scaled sizes. The decoupled kinematics of the outer and inner discs are represented by the blue and red colours, which depict the orientation of the blue-shifted and red-shifted rotating gas. The rotation direction is indicated by the curved arrows. The intensity of the colours illustrate the velocities; the inner disc rotates at a higher speed than the outer disc. The stars in the inner disc aim to show the region where most of the starburst is located. The radial arrows represent the low-velocity expansion of the core. 
Table 5. Brightness temperature ratios in Mrk 273 (row/column), evaluated over the entire emission.

\begin{tabular}{|c|c|c|c|c|c|c|c|}
\hline & $\mathrm{HCN}(1-0)$ & $\mathrm{HCO}^{+}(1-0)$ & $\mathrm{HNC}(1-0)$ & $\mathrm{HC}_{3} \mathrm{~N}(10-9)$ & $\mathrm{HCN}(3-2)$ & $\mathrm{HCO}^{+}(3-2)$ & $\mathrm{HOC}^{+}(3-2)^{c}$ \\
\hline $\operatorname{HCN}(1-0)$ & $1.00 \pm 0.07$ & $1.00 \pm 0.07$ & $1.8 \pm 0.2$ & $6 \pm 1$ & - & - & - \\
\hline $\mathrm{HCO}^{+}(1-0)$ & $1.00 \pm 0.07$ & $1.00 \pm 0.07$ & $1.8 \pm 0.2$ & $6 \pm 1$ & _- & - & _- \\
\hline $\mathrm{HNC}(1-0)$ & $0.55 \pm 0.05$ & $0.55 \pm 0.05$ & $1.0 \pm 0.1$ & $3.2 \pm 0.8$ & - & - & - \\
\hline $\mathrm{HC}_{3} \mathrm{~N}(10-9)$ & $0.17 \pm 0.04$ & $0.17 \pm 0.04$ & $0.31 \pm 0.08$ & $1.0 \pm 0.3$ & - & - & - \\
\hline $\operatorname{HCN}(3-2)$ & - & - & - & - & $1.00 \pm 0.06$ & $1.13 \pm 0.06$ & $10 \pm 5$ \\
\hline $\mathrm{HCO}^{+}(3-2)$ & - & - & - & - & $0.88 \pm 0.05$ & $1.00 \pm 0.06$ & $9 \pm 4$ \\
\hline $\mathrm{HOC}^{+}(3-2)^{a}$ & - & - & - & - & $0.10 \pm 0.05$ & $0.11 \pm 0.06$ & $1.0 \pm 0.7$ \\
\hline
\end{tabular}

Notes. Due to differences in the observed areas, we do not list the ratios between the $3 \mathrm{~mm}$ and $1 \mathrm{~mm}$ lines (see Sect. 4.5$)$. ${ }^{(a)} \mathrm{For} \mathrm{HOC}^{+}(3-2)$, we used the temperature measured in the pixel where it peaks (see Sect. 3.2.1).

Table 6. Nuclear brightness temperature ratios in Mrk 273 (row/column), evaluated in the central pixel.

\begin{tabular}{|c|c|c|c|c|c|c|c|}
\hline & $\operatorname{HCN}(1-0)$ & $\mathrm{HCO}^{+}(1-0)$ & HNC $(1-0)$ & $\mathrm{HC}_{3} \mathrm{~N}(10-9)$ & $\operatorname{HCN}(3-2)$ & $\mathrm{HCO}^{+}(3-2)$ & $\mathrm{HOC}^{+}(3-2)^{a}$ \\
\hline $\operatorname{HCN}(1-0)$ & $1.00 \pm 0.07$ & $1.03 \pm 0.07$ & $2.2 \pm 0.2$ & $9 \pm 2$ & - & - & - \\
\hline $\mathrm{HCO}^{+}(1-0)$ & $0.97 \pm 0.06$ & $1.00 \pm 0.07$ & $2.1 \pm 0.2$ & $9 \pm 2$ & - & - & - \\
\hline $\mathrm{HNC}(1-0)$ & $0.45 \pm 0.04$ & $0.46 \pm 0.04$ & $1.0 \pm 0.1$ & $4 \pm 1$ & - & - & - \\
\hline $\mathrm{HC}_{3} \mathrm{~N}(10-9)$ & $0.11 \pm 0.02$ & $0.12 \pm 0.03$ & $0.25 \pm 0.06$ & $1.0 \pm 0.3$ & - & - & - \\
\hline $\mathrm{HCN}(3-2)$ & - & - & - & - & $1.00 \pm 0.06$ & $1.28 \pm 0.08$ & $7 \pm 3$ \\
\hline $\mathrm{HCO}^{+}(3-2)$ & - & - & - & - & $0.78 \pm 0.05$ & $1.00 \pm 0.07$ & $5 \pm 3$ \\
\hline $\mathrm{HOC}^{+}(3-2)^{a}$ & - & - & - & - & $0.14 \pm 0.07$ & $0.18 \pm 0.09$ & $1.0 \pm 0.7$ \\
\hline
\end{tabular}

Notes. Due to differences in the observed areas, we do not list the ratios between the $3 \mathrm{~mm}$ and $1 \mathrm{~mm}$ lines (see Sect. 4.5$)$. ${ }^{(a)} \mathrm{For} \mathrm{HOC}^{+}(3-2)$ we used the temperature measured in the pixel where it peaks (see Sect. 3.2.1).

Table 7. Main parameters of the undetected vibrational transitions of $\mathrm{HCN}$ and $\mathrm{HC}_{3} \mathrm{~N}$.

\begin{tabular}{lccccccc}
\hline \hline Line & $\begin{array}{c}\text { Freq } \\
(\mathrm{GHz})\end{array}$ & $\begin{array}{c}E_{\text {low }} \\
(\mathrm{K})\end{array}$ & $\begin{array}{c}A_{\mathrm{ul}} \\
\left(\times 10^{-3} \mathrm{~s}^{-1}\right)\end{array}$ & $\begin{array}{c}n_{\text {crit }} \\
\left(\mathrm{cm}^{-3}\right)\end{array}$ & $\begin{array}{c}\text { Flux } \\
\left(\mathrm{K} \mathrm{km} \mathrm{s}^{-1}\right)\end{array}$ & $\begin{array}{c}\text { Line Peak } \\
(\mathrm{mJy})\end{array}$ & $\begin{array}{c}L^{\prime} \\
\left(\mathrm{K} \mathrm{km} \mathrm{s}^{-1} \mathrm{pc}^{2}\right)\end{array}$ \\
\hline $\mathrm{HC}_{3} \mathrm{~N}(10-9) v_{6}=1$ & 91.202 & 512 & 0.15 & $>10^{11}$ & $\leq 1.1$ & $\leq 0.3$ & $\leq 6.4 \times 10^{6}$ \\
$\mathrm{HCN}(3-2) v_{2}=1$ & 267.199 & 720 & 0.73 & $>10^{10}$ & $\leq 3.7$ & $\leq 1.1$ & $\leq 7.3 \times 10^{5}$ \\
\hline
\end{tabular}

(i.e. $\mathrm{HCN} / \mathrm{HNC} \gg 1$ ) being associated with shock-dominated regions, which are common in early starbursts (Aladro et al. 2015).

The brightness temperature ratio between $\operatorname{HCN}(1-0)$ and $\mathrm{HNC}(1-0)$ in Mrk 273 is 1.7 (2.1 in the central pixel). Previous observations of these lines with the OSO and SEST single-dish telescopes yielded an integrated intensity ratio $\geq 4$ (Aalto et al. 2002), although that value is based on an upper limit to the HNC line. Taking our result of 1.7 as the minimum value, it is safe to say that this conservative ratio is moderately high, and that Mrk 273 cannot be classified as an HNC-luminous galaxy.

As discussed above (Sect. 4.5.1), the $\mathrm{HCN} / \mathrm{HCO}^{+}$brightness temperature ratio we obtain for Mrk 273 does not allow us to favour a starburst over an AGN-dominated nucleus. The HNC and $\mathrm{HCO}^{+}$intensities appear to be anti-correlated in (U)LIRGs, as observed by Costagliola et al. (2011) in a fairly large sample of galaxies. From our data, we obtain an $\mathrm{HCO}^{+} / \mathrm{HNC}=1.8 \pm 0.2$ (Table 6). If the northern nucleus of Mrk 273 is dominated by a starburst (as claimed by Condon et al. 1991; Majewski et al. 1993; Downes \& Solomon 1998), the low HNC abundance would indicate that the gas comes from warm and dense phases in an early stage, and would explain why $\mathrm{HCO}^{+}$is relatively abundant. However, models by Rodríguez Zaurín et al. (2009) indicate that most of the stellar population in Mrk 273 has an age of $0.7-10 \mathrm{Gyr}$ (although there might be a significant fraction of stars younger than $50 \mathrm{Myr}$ ), which challenges an early starburst scenario. In the case of an AGN (as claimed by U et al. 2013; Rodríguez Zaurín et al. 2014; Iwasawa et al. 2018), HNC does not necessarily need to be faint, but HCN could be boosted. However, in the latter scenario one would expect a higher $\mathrm{HCN} / \mathrm{HCO}^{+}$ratio.

\subsubsection{An extremely low $\mathrm{HCO}^{+} / \mathrm{HOC}^{+}$ratio}

$\mathrm{HOC}^{+}$, the metastable isomer of $\mathrm{HCO}^{+}$, is efficiently formed via the following ion-molecule reactions;

$$
\begin{aligned}
& \mathrm{CO}^{+}+\mathrm{H}_{2} \rightarrow \mathrm{HOC}^{+}+\mathrm{H} \\
& \mathrm{C}^{+}+\mathrm{H}_{2} \mathrm{O} \rightarrow \mathrm{HOC}^{+}+\mathrm{H} \\
& \mathrm{H}_{3}^{+}+\mathrm{CO} \rightarrow \mathrm{HOC}^{+}+\mathrm{H}_{2}
\end{aligned}
$$

and is mainly destroyed by reactions with $\mathrm{H}_{2}$ (Jarrold et al. 1986; Smith et al. 2002; Fuente et al. 2003):

$\mathrm{HOC}^{+}+\mathrm{H}_{2} \rightarrow \mathrm{HCO}^{+}+\mathrm{H}_{2}$.

While typical values of the $\mathrm{HCO}^{+} / \mathrm{HOC}^{+}$ratio in Galactic dense molecular clouds range between 300 and 6000 (Apponi \& Ziurys 1997), values as low as 50-150 are found in several Galactic and extragalactic PDRs and XDRs (X-ray 


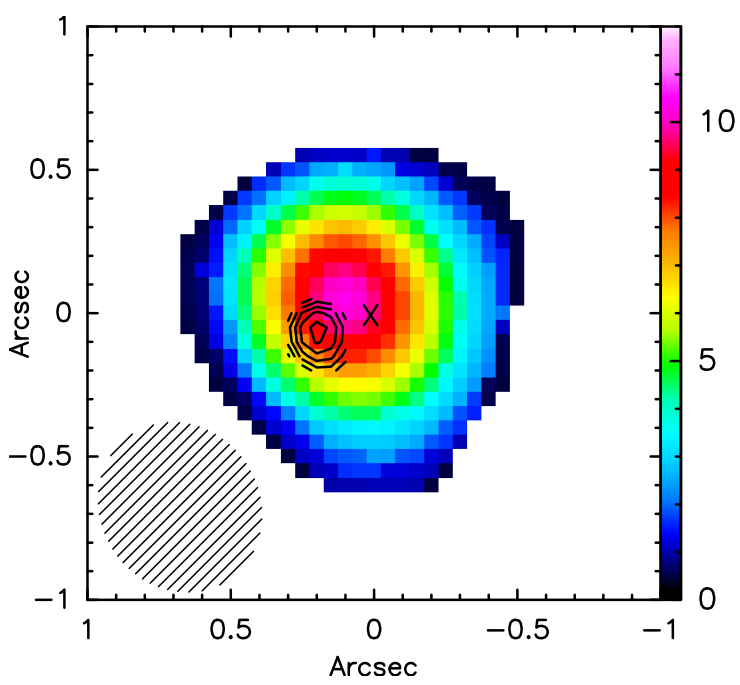

Fig. 17. $\mathrm{HCO}^{+}(3-2)$ blue-shifted intensity colours (in units of Jy km s${ }^{-1}$ beam ${ }^{-1}$ ) integrated from $-300 \mathrm{~km} \mathrm{~s}^{-1}$ to $0 \mathrm{~km} \mathrm{~s}^{-1}$ with $\operatorname{HOC}^{+}(3-2)$ contours (starting from $3 \sigma=3.3 \mathrm{mJy}^{-1}$ beam ${ }^{-1}$ ) over-plotted with steps of $10 \mathrm{mJy}$ beam $^{-1}$. The cross at the centre marks the continuum peak. The synthesised beam is shown in the bottom-left corner.

dominated regions), likely as a consequence of high ionisation rates created by ionisation fields (UV, cosmic rays, and/or $\mathrm{X}$-ray radiation; Fuente et al. 2003; Usero et al. 2004). From our observations, we derive a global brightness temperature ratio $\mathrm{HCO}^{+}(3-2) / \mathrm{HOC}^{+}(3-2)=9 \pm 4$, and a nuclear ratio of $5 \pm 3$. Such low values have only been found in other ULIRGs hosting extremely compact obscured nuclei, namely IC 860, Zw 049.057, and Mrk 231 (Aalto et al. 2015a,b). The physical and chemical reasons for these low ratios are still not known and merit further study, but high opacities of $\mathrm{HCO}^{+}$could be responsible.

\subsection{A different origin of $\mathrm{HOC}^{+}$emission}

As mentioned in Sect. 3.3, $\operatorname{HOC}^{+}(3-2)$ peaks at $\mathrm{RA}(\mathrm{J} 2000)=$ $13^{\mathrm{h}} 44^{\mathrm{m}} 42^{\mathrm{s}} \cdot 15, \operatorname{Dec}(\mathrm{J} 2000)=55^{\circ} 53^{\prime} 13^{\prime \prime} \cdot 45$, which corresponds to an offset $\left(0^{\prime \prime} \cdot 2,-0^{\prime \prime} .05\right)$ south-east of the central pixel. This indicates that its emission has a different origin from the rest of the dense gas tracers. Furthermore, the two $\mathrm{HOC}^{+}$components are blue-shifted in velocity with respect to $\mathrm{HCN}$ and $\mathrm{HCO}^{+}(3-2)$ (Table 1). Figure 17 shows the $\mathrm{HOC}^{+}$emission in contours plotted over the blue-shifted $\mathrm{HCO}^{+}$emission integrated between -300 and $0 \mathrm{~km} \mathrm{~s}^{-1}$. This plot shows that $\mathrm{HOC}^{+}$does not peak at the same position as the blue-shifted dense gas, and that there is not even detected emission of the species in the central pixel.

Why is the emission of this species shifted with respect to the others? One possibility is that the central pixels are heavily obscured and $\mathrm{HOC}^{+}$is completely absorbed there. However, in that case one would expect some kind of symmetric emission around the nucleus, with a ring-like shape, or at least other peaks of emission around the nucleus, which are not seen. This strongly suggests that $\mathrm{HOC}^{+}$is not peaking at the very centre.

We checked the literature looking for $\mathrm{OH}$ masers, supernovae, radio continuum sources, or any source emitting at the $\mathrm{HOC}^{+}$peak coordinates. High-angular resolution NIR observations conducted by $\mathrm{U}$ et al. (2013) resolve the northern nucleus of Mrk 273 into three components, called N1, N2, and N3. Component $\mathrm{N} 1$ is the brightest of the three and is associated with the true nucleus (our $\left(0^{\prime \prime}, 0^{\prime \prime}\right)$ position). N3 is found $\sim 0^{\prime \prime}$. 15 to the south-east of $\mathrm{N} 1$, very near the $\mathrm{HOC}^{+}$maximum flux density. The peak of the $\mathrm{HOC}^{+}$emission might correspond to $\mathrm{N} 3$, but better astrometry is needed in order to strengthen this association. The nature of $\mathrm{N} 3$ is, in any case, not clear, but it does have stronger emission from [FeII] than N1. It could be a separated clump, a compact star cluster, or a supernova remnant. N3 does not appear in radio continuum maps (Carilli \& Taylor 2000; Bondi et al. 2005), leaving a supernova remnant as a less likely option. To further address the origin of this species, deeper highresolution observations of $\mathrm{HOC}^{+}$would be needed to determine its extent and to more accurately measure its position.

\section{Conclusions}

We have used the NOEMA interferometer to observe several spectral lines of $\mathrm{HCN}, \mathrm{HCO}^{+}, \mathrm{HOC}^{+}, \mathrm{HNC}$, and $\mathrm{HC}_{3} \mathrm{~N}$ with angular resolutions of $\left(4^{\prime \prime} .9 \times 4^{\prime \prime} .5\right)$ and $\left(0^{\prime \prime} .61 \times 00^{\prime \prime} .55\right)$ (corresponding to spatial scales of $\sim(3.7 \times 3.4) \mathrm{kpc}$ and $\sim(460 \times 420) \mathrm{pc})$. We also included multiple lines of $\mathrm{H}_{2} \mathrm{O}$ observed with the Herschel SPIRE and PACS instruments. Our observations, extending from the $\mathrm{mm}$ to the FIR regime, allowed us to study the properties of the gaseous disc in the northern nucleus of Mrk 273, as well as the connection between its cold and warm phases. We summarise the main results as follows.

Morphology and kinematics of the disc. The cold and dense gas in the nuclear disc (traced by $\mathrm{HCN}$ and $\mathrm{HCO}^{+}$) has two components with decoupled kinematics. The low-excitation gas in the outer parts of the disc extends up to a radius of $\sim 1.5 \mathrm{kpc}$ and rotates from south-east to north-west (with a PA of approximately $-40^{\circ}$ ), while the more excited dense gas arising from the central star forming region $(<300 \mathrm{pc})$ is characterised by a north-east to south-west rotation $\left(\mathrm{PA} \sim 70^{\circ}\right)$. This inner disc contains a dynamical mass of $3 \times 10^{9} \mathrm{M}_{\odot}$, and a luminosity of $L_{\mathrm{HCN}}^{\prime}=3 \times 10^{8} \mathrm{~K} \mathrm{~km} \mathrm{~s}^{-2} \mathrm{pc}^{2}$. The warm gas, traced by the FIR $\mathrm{H}_{2} \mathrm{O}$ lines, can also be separated into two components: a warm and very compact core with a radius of $\sim 50 \mathrm{pc}$ and a temperature of $95 \mathrm{~K}$, and a more extended and relatively cooler component, with $R<300 \mathrm{pc}$ and $T=55 \mathrm{~K}$.

The extended component of the warm gas and the compact component of the cold gas are co-spatial in the inner $\sim 300 \mathrm{pc}$. The $\mathrm{H}_{2}$ column densities and dust properties obtained from our water modelling, as well as the line profiles of the dense gas tracers, show that this region is significantly affected by dust obscuration. The blue-shifted emission of the bulk of gas (consistently seen in all observed lines) also indicates that the core is expanding outwards at low velocities $\left(v-v_{\text {sys }} \sim 50-100 \mathrm{~km} \mathrm{~s}^{-1}\right)$, likely affected by the outflow.

Outflow properties. We detected the cold (sub-mm) and warm (FIR) phases of the Mrk 273 molecular outflow. It is a compact outflow, being expelled to distances of $\sim 460 \mathrm{pc}$ mostly towards the northern direction, but it reaches high velocities of $\sim 1000 \mathrm{~km} \mathrm{~s}^{-1}$. This fast outflow $\left(\left|v-v_{\text {sys }}\right|>400 \mathrm{~km} \mathrm{~s}^{-1}\right)$ has a luminosity of $8 \times 10^{7} \mathrm{~K} \mathrm{~km} \mathrm{~s}^{-1} \mathrm{pc}^{2}$, and a mass of dense gas $M_{\text {dense }}^{\text {outlow }} \leq 8 \times 10^{8} M_{\odot}$. The difference in PA between the major kinematic axis of the inner disc $\left(71^{\circ} \pm 5^{\circ}\right)$ and that of the outflow $\left(10^{\circ} \pm 3^{\circ}\right)$ suggests that the latter is probably driven by the AGN.

Chemistry. We explored the chemistry of Mrk 273 by means of molecular line ratios. The most outstanding ratio is that of $\mathrm{HCO}^{+} / \mathrm{HOC}^{+}$. We estimated it to be $<10$, one of the lowest values ever measured in any galactic or extragalactic source. The reason for this value, however, is still not clear and should be 
further studied in detail with the help of chemical models. It is worth noting, however, that the origin of $\mathrm{HOC}^{+}$is different from the rest of the detected molecular species, since its emission is spatially shifted from the centre.

Regarding the outflow, our non-detection of the highvelocity wind in $\mathrm{HCO}^{+}$, together with the non-detection of the red-shifted outflowing gas either in $\mathrm{HCN}$ and $\mathrm{HCO}^{+}$, suggests the possibility of chemical differentiation. However, we note that, despite our high sensitivity, the fast outflow of Mrk 273 is very faint, meaning that deeper observations would be necessary to better probe its chemistry.

Acknowledgements. This work is based on observations carried out under project numbers W14DD and E16AK with the IRAM NOEMA Interferometer. IRAM is supported by INSU/CNRS (France), MPG (Germany) and IGN (Spain). The research leading to these results has received funding from the European Union's Horizon 2020 research and innovation program under grant agreement No 730562 [RadioNet]. This research has made use of NASA's Astrophysics Data System, and the NASA/IPAC Extragalactic Database (NED), which is operated by the Jet Propulsion Laboratory, California Institute of Technology, under contract with the National Aeronautics and Space Administration. We are grateful to the referee for the fast and constructive report, as well as to the IRAM/NOEMA staff for their help during the observations and data reduction. RA would like to thank Leslie Hunt and Loreto Barcos-Muñoz for the useful discussions about Mrk 273.

\section{References}

Aalto, S., Polatidis, A. G., Hüttemeister, S., \& Curran, S. J. 2002, A\&A, 381, 783

Aalto, S., Monje, R., \& Martín, S. 2007, A\&A, 475, 479

Aalto, S., Garcia-Burillo, S., Muller, S., et al. 2012, A\&A, 537, A44

Aalto, S., Garcia-Burillo, S., Muller, S., et al. 2015a, A\&A, 574, A85

Aalto, S., Martín, S., Costagliola, F., et al. 2015b, A\&A, 584, A42

Aalto, S., Muller, S., Costagliola, F., et al. 2017, A\&A, 608, A22

Aladro, R., Martín-Pintado, J., Martín, S., Mauersberger, R., \& Bayet, E. 2011, A\&A, 525, A89

Aladro, R., Viti, S., Bayet, E., et al. 2013, A\&A, 549, A39

Aladro, R., Martín, S., Riquelme, D., et al. 2015, A\&A, 579, A101

Apponi, A. J., \& Ziurys, L. M. 1997, ApJ, 481, 800

Armus, L., Charmandaris, V., Bernard-Salas, J., et al. 2007, ApJ, 656, 148

Bondi, M., Pérez-Torres, M.-A., Dallacasa, D., \& Muxlow, T. W. B. 2005, MNRAS, 361, 748

Botinelli, L., Fraix-Burnet, D., Gouguenheim, L., Le Squeren, A. M., \& Patey, I. 1985, IAU Circ., 4074, 1

Carilli, C. L., \& Taylor, G. B. 2000, ApJ, 532, L95

Cicone, C., Maiolino, R., Sturm, E., et al. 2014, A\&A, 562, A21

Cole, G. H. J., Pedlar, A., Holloway, A. J., \& Mundell, C. G. 1999, MNRAS, 310,1033

Colina, L., Arribas, S., \& Borne, K. D. 1999, ApJ, 527, L13

Condon, J. J. 1997, PASP, 109, 166

Condon, J. J., Huang, Z.-P., Yin, Q. F., \& Thuan, T. X. 1991, ApJ, 378, 65

Costagliola, F., \& Aalto, S. 2010, A\&A, 515, A71

Costagliola, F., Aalto, S., Rodriguez, M. I., et al. 2011, A\&A, 528, A30

Costagliola, F., Sakamoto, K., Muller, S., et al. 2015, A\&A, 582, A91

Daniel, F., Dubernet, M.-L., \& Grosjean, A. 2011, A\&A, 536, A76

Davies, R. I., Tacconi, L. J., \& Genzel, R. 2004, ApJ, 613, 781

Downes, D., \& Solomon, P. M. 1998, ApJ, 507, 615

Dubernet, M.-L., Daniel, F., Grosjean, A., \& Lin, C. Y. 2009, A\&A, 497, 911

Falstad, N., González-Alfonso, E., Aalto, S., \& Fischer, J. 2017, A\&A, 597, A105
Fuente, A., Rodríguez-Franco, A., García-Burillo, S., Martín-Pintado, J., \& Black, J. H. 2003, A\&A, 406, 899

Gao, Y., \& Solomon, P. M. 2004, ApJS, 152, 63

Gao, Y., Gruendl, R. A., Hwang, C.-Y., \& Lo, K. Y. 1999, Galaxy Interactions at Low and High Redshift, 186, 227

García-Burillo, S., Combes, F., Usero, A., et al. 2015, A\&A, 580, A35

González-Alfonso, E., \& Cernicharo, J. 1997, A\&A, 322, 938

González-Alfonso, E., \& Cernicharo, J. 1999, ApJ, 525, 845

González-Alfonso, E., Fischer, J., Isaak, K., et al. 2010, A\&A, 518, L43

González-Alfonso, E., Fischer, J., Aalto, S., \& Falstad, N. 2014, A\&A, 567, A91

González-Alfonso, E., Fischer, J., Spoon, H. W. W., et al. 2017, ApJ, 836, 11

Graciá-Carpio, J., García-Burillo, S., Planesas, P., Fuente, A., \& Usero, A. 2008, A\&A, 479, 703

Griffin, M. J., Abergel, A., Abreu, A., et al. 2010, A\&A, 518, L3

Huettemeister, S., Henkel, C., Mauersberger, R., et al. 1995, A\&A, 295, 571

Imanishi, M., Nakanishi, K., \& Kohno, K. 2006, AJ, 131, 2888

Imanishi, M., Nakanishi, K., Tamura, Y., \& Peng, C.-H. 2009, AJ, 137, 3581

Imanishi, M., Nakanishi, K., \& Izumi, T. 2016, AJ, 152, 218

Ivison, R. J., Greve, T. R., Dunlop, J. S., et al. 2007, MNRAS, 380, 199

Iwasawa, K., U, V., Mazzarella, J. M., et al. 2018, A\&A, 611, A71

Izumi, T., Kohno, K., Martín, S., et al. 2013, PASJ, 65, 100

Izumi, T., Kohno, K., Aalto, S., et al. 2016, ApJ, 818, 42

Janssen, A. W., Christopher, N., Sturm, E., et al. 2016, ApJ, 822, 43

Jarrold, M. F., Bowers, M. T., Defrees, D. J., McLean, A. D., \& Herbst, E. 1986, ApJ, 303, 392

Kohno, K., Matsushita, S., Vila-Vilaró, B., et al. 2001, The Central Kiloparsec of Starbursts and AGN: The La Palma Connection, 249, 672

Krips, M., Neri, R., García-Burillo, S., et al. 2008, ApJ, 677, 262

Lahuis, F., Spoon, H. W. W., Tielens, A. G. G. M., et al. 2007, ApJ, 659, 296

Lindberg, J. E., Aalto, S., Muller, S., et al. 2016, A\&A, 587, A15

Lu, N., Zhao, Y., Díaz-Santos, T., et al. 2017, ApJS, 230, 1

Majewski, S. R., Hereld, M., Koo, D. C., Illingworth, G. D., \& Heckman, T. M. 1993, ApJ, 402, 125

Martín, S., Mauersberger, R., Martín-Pintado, J., Henkel, C., \& García-Burillo, S. 2006, ApJS, 164, 450

Martín, S., Krips, M., Martín-Pintado, J., et al. 2011, A\&A, 527, A36

Martín, S., Kohno, K., Izumi, T., et al. 2015, A\&A, 573, A116

Martín, S., Aalto, S., Sakamoto, K., et al. 2016, A\&A, 590, A25

Medling, A. M., U, V., Guedes, J., et al. 2014, ApJ, 784, 70

Meijerink, R., Spaans, M., \& Israel, F. P. 2006, ApJ, 650, L103

Nardini, E., Risaliti, G., Salvati, M., et al. 2009, MNRAS, 399, 1373

Ott, S. 2010, Astronomical Data Analysis Software and Systems XIX, 434, 139

Pickett, H. M., Poynter, R. L., Cohen, E. A., et al. 1998, J. Quant. Spectr. Rad. Transf., 60, 883

Poglitsch, A., Waelkens, C., Geis, N., et al. 2010, A\&A, 518, L2

Privon, G. C., Herrero-Illana, R., Evans, A. S., et al. 2015, ApJ, 814, 39

Rangwala, N., Maloney, P. R., Wilson, C. D., et al. 2015, ApJ, 806, 17

Rigopoulou, D., Lawrence, A., \& Rowan-Robinson, M. 1996, MNRAS, 278,1049

Rodríguez Zaurín, J., Tadhunter, C. N., \& González Delgado, R. M. 2009. MNRAS, 400, 1139

Rodríguez Zaurín, J., Tadhunter, C. N., Rupke, D. S. N., et al. 2014, A\&A, 571, A57

Sakamoto, K., Aalto, S., Evans, A. S., Wiedner, M. C., \& Wilner, D. J. 2010, ApJ, 725, L228

Savage, C., \& Ziurys, L. M. 2004, ApJ, 616, 966

Smith, M. A., Schlemmer, S., von Richthofen, J., \& Gerlich, D. 2002, ApJ, 578, L87

Toomre, A. 1964, ApJ, 139, 1217

U, V., Medling, A., Sanders, D., et al. 2013, ApJ, 775, 115

Usero, A., García-Burillo, S., Fuente, A., Martín-Pintado, J., \& RodríguezFernández, N. J. 2004, A\&A, 419, 897

Veilleux, S., Rupke, D. S. N., Kim, D.-C., et al. 2009, ApJS, 182, 628

Veilleux, S., Meléndez, M., Sturm, E., et al. 2013, ApJ, 776, 27

Wilson, C. D., et al. 2008, ApJS, 178, 189

Yun, M. S., \& Scoville, N. Z. 1995, ApJ, 451, L45 